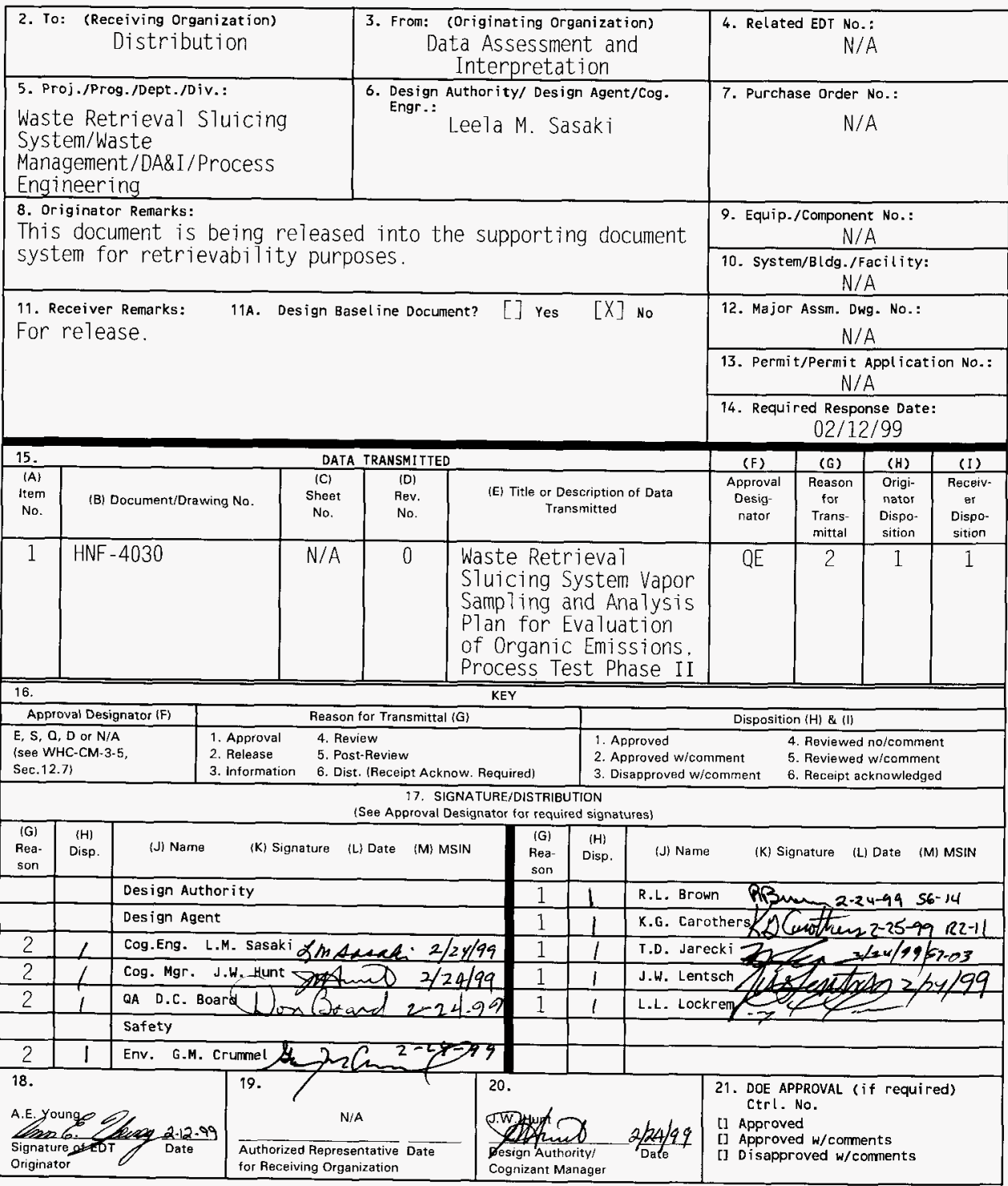




\title{
Waste Retrieval Sluicing System Vapor Sampling and Analysis Plan for Evaluation of Organic Emissions, Process Test Phase II
}

Leela M. Sasaki

Lockheed Martin Hanford, Corp. , Richland. WA 99352

U.S. Department of Energy Contract DE-AC06-96RL13200

\author{
EDT/ECN: EDT-611450 UC: 2070 \\ Org Code: 74B20 Charge Code: CACN 101997/COA BA10 \\ B\&R Code: EW 3120074 Total Pages: 34
}

Key Words: Waste Retrieval Sluicing System, WRSS, Vapor. Sampling,

Analysis. Plan. Retrieval Project, Characterization, Retrieval.

Evaluation, Organic Emissions, Organic, Emission. Process Test, Phase 2

Abstract: N/A

TRADEMARK DISCLAIMER. Reference herein to any specific commercial product, process, or service by trade name, trademark, manufacturer, or otherwise, does not necessarily constitute or imply its endorsement, recommendation, or favoring by the United States Government or any agency thereof or its contractors or subcontractors.

Printed in the United states of America. To obtain copies of this document, contact: Document Control Services, P.O. Box 950, Mailstop H6-08, Richland WA 99352, Phone (509) 372-2420;

Fax (509) 376-4989.
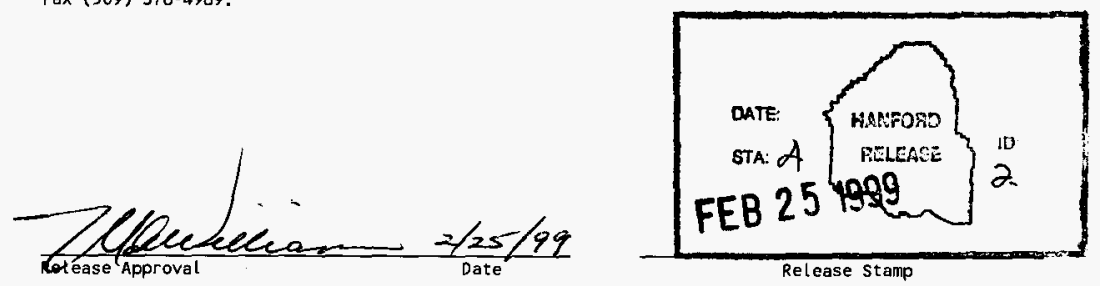

\section{Approved for Public Release}




\section{Waste Retrieval Sluicing System Vapor Sampling and Analysis Plan for Evaluation of Organic Emissions, Process Test Phase II}

L. M. Sasaki

Lockheed Martin Hanford Corporation

Prepared for the U.S. Department of Energy Office of Environmental Restoration and Waste Management 
HNF-4030, Rev. 0

\section{TABLE OF CONTENTS}

1.0 SAMPLING AND ANALYSIS OBJECTIVES $\ldots \ldots \ldots \ldots \ldots \ldots$

2.0 SAMPLING EVENT REQUIREMENTS $\ldots \ldots \ldots \ldots \ldots \ldots \ldots \ldots \ldots \ldots \ldots$

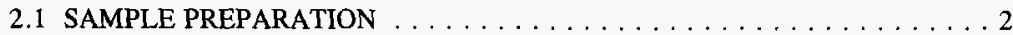

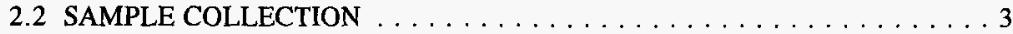

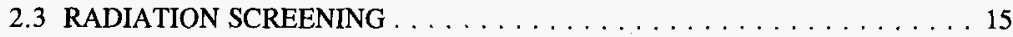

3.0 LABORATORY ANALYSIS REQUIREMENTS $\ldots \ldots \ldots \ldots \ldots \ldots$

4.0 QUALITY ASSURANCE AND QUALITY CONTROL $\ldots \ldots \ldots \ldots \ldots \ldots \ldots 22$

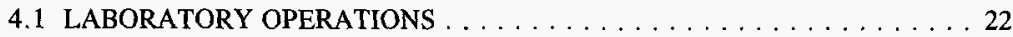

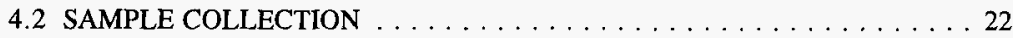

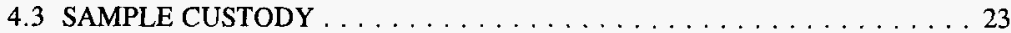

5.0 EXCEPTIONS, CLARIFICATIONS, AND ASSUMPTIONS $\ldots \ldots \ldots \ldots 24$

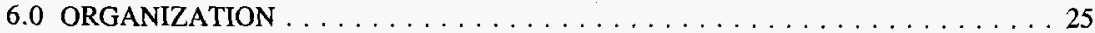

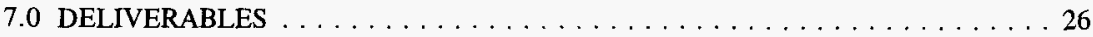

7.1 FORMAT I REPORTING $\ldots \ldots \ldots \ldots \ldots \ldots \ldots \ldots \ldots \ldots \ldots \ldots$

7.2. FORMAT II REPORTING $\ldots \ldots \ldots \ldots \ldots \ldots \ldots \ldots \ldots \ldots \ldots$

7.3 FORMAT VI REPORTING $\ldots \ldots \ldots \ldots \ldots \ldots \ldots \ldots \ldots \ldots \ldots$

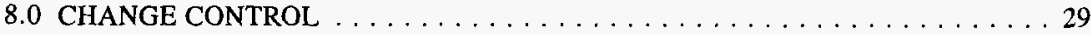

9.0 REFERENCES . . . . . . . . . . . . . . . . . . . . . . 30

\section{LIST OF TABLES}

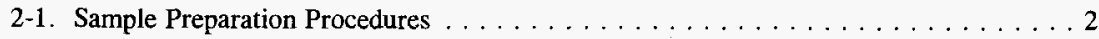

2-2. List of Samples and Activities for 296-C-006 Stack . . . . . . . . . . . . . . 5

2-3. List of Samples and Activities for Ambient Air Sampling in C Tank Farm . . . . . . 12

2-4. List of Samples and Activities for 241-AZ-702 Stack . . . . . . . . . . . 13

2-5. Vapor Sampling Summary . . . . . . . . . . . . . . . . . . . 14

3-1. Chemical and Radiological Analytical Requirements . . . . . . . . . . . 17

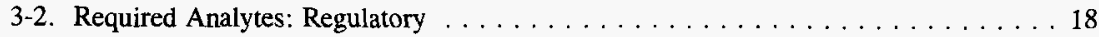

3-3. Required Analytes: Program Requested Analytes/Selected Compounds Observed in Tank 241-C-106 Vapor or Waste Samples . . . . . . . . . . . . . . . . . . 19

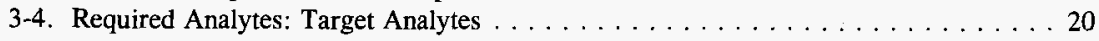

6-1. Project Key Personnel . . . . . . . . . . . . . . . . . . . . . . . . . 25

7-1. Data Package Required Elements . . . . . . . . . . . . . . . . 27 
HNF-4030, Rev. 0

\section{LIST OF ABBREVIATIONS}

CAS Chemical Abstracts Service

CPO Characterization Project Operations

DBP dibutyl phosphate

FID flame ionization detector

GC/MS gas chromatography/mass spectrometry

GC/TCD gas chromatography/thermal conductivity detector

HASQARD Hanford Analytical Services Quality Assurance Requirements Document

IC ion chromatography

IDLH immediately dangerous to life and health

LFL lower flammability limit

LMHC Lockheed Martin Hanford Corporation

N/A not applicable or not available

NEVS non-electrical sampling system

NHC Numatec Hanford Corporation

ppmv parts per million by volume

PUF polyurethane foam

QA quality assurance

QC quality control

SAS Special Analytical Support

STT sorbent tube train

TBP tributyl phosphate

TOC total organic carbon

TST triple sorbent trap

TWRS Tank Waste Remediation System

WSCF Waste Sampling and Characterization Facility 
HNF-4030, Rev. 0

\subsection{SAMPLING AND ANALYSIS OBJECTIVES}

This sampling and analysis plan identifies characterization objectives pertaining to sample collection, laboratory analytical evaluation, and reporting requirements for vapor samples obtained to address vapor issued related to the sluicing of tank 241-C-106. Sampling will be performed in accordance with Waste Retrieval Sluicing System Emissions Collection Phase II (Jones 1999) and Process Test Plan Phase II, Waste Retrieval Sluicing System Emissions Collection (Powers 1999). Analytical requirements include those specified in Request for Ecology Concurrence on Draft Strategy/Path Forward to Address Concerns Regarding Organic Emissions from C-106 Sluicing Activities (Peterson 1998).

The Waste Retrieval Sluicing System was installed to retrieve and transfer high-heat sludge from tank 241-C-106 to tank 241-AY-102, which is designed for high-heat waste storage. During initial sluicing of tank 241-C-106 in November 1998, operations were halted due to detection of unexpected high total organic compounds that exceeded regulatory permit limits. Several workers also reported smelling sharp odors and throat irritation. Vapor grab samples from the 296-C-006 ventilation system were taken as soon as possible after the detection; the analyses indicated that volatile and semi-volatile organic compounds were present.

In December 1998, a process test (phase I) was conducted in which the pumps in tanks 241-C106 and 241-AY-102 were operated and vapor samples obtained to determine constituents that may be present during active sluicing of tank 241-C-106. The process test was suspended when a jumper leak was detected. A goal of phase II of the process test will be to obtain representative vapor samples at higher concentrations than those achieved in phase I of the process test. During phase II of the process test, vapor samples will be obtained from the 296C-006 ventilation system stack and 241-AZ-702 ventilation system stack. Ambient air samples will also be obtained at several locations. Results will be used to address the following:

- $\quad$ Provide gas composition data that can be used to determine personnel protective measures,

- $\quad$ Provide gas composition data that can be used for control equipment selection and design, if required,

- Substantiate that ammonia and organic emissions are below regulatory thresholds during the test.

The following sections provides the general methodology and procedures to be used in the preparation, retrieval, transport, analysis, and reporting of results from vapor samples retrieved during the process test. 


\subsection{SAMPLING EVENT REQUIREMENTS}

The waste retrieval sluicing system will be operated in accordance with the process test plan and process test procedure (Powers 1999, Jones 1999) to raise the total organic carbon (TOC) concentration in the 296-C-006 ventilation stack to levels above those reached in the phase I of the process test. It is anticipated that stack TOC levels of approximately $300-400$ parts per million by volume (ppmv) will be reached. Initially during the test, the effects of sluicer jet direction on the release of volatile organic compounds will be tested. Based on this information, waste agitation will be controlled above $300 \mathrm{ppmv}$. The pumps will be tripped at $400 \mathrm{ppmv}$. Emergency evacuation will be conducted at 500 ppmv. The effects of the ventilation recirculation heater may be tested. Sluicing duration for the performance of this testing is limited to approximately 20 hours ( 9 hours maximum for the next sluice batch and the balance of the operating time for the subsequent batch). Vapor samples will be taken prior to, during, and after pump operation.

\subsection{SAMPLE PREPARATION}

SUMMA ${ }^{1}$ canister samples, triple sorbent trap (TST) samples, sorbent tube train (STT) samples, and polyurethane foam (PUF) samples will be collected and sent to the laboratory for analysis; field and trip blanks will accompany the samples. The analytical laboratory for this sampling event is Numatec Hanford Corporation (NHC) Special Analytical Support (SAS). Particulate filter samples will be collected for radiation screening of the samples. SAS shall prepare the SUMMA canisters, TSTs, STTs, and PUFs for sample collection.

Characterization Project Operations (CPO) shall prepare the particulate filter assemblies for use at the 241-AZ-702 stack and SAS shall prepare the particulate filter assemblies for use at the 296-C-006 stack; each particulate filter assembly shall contain two particulate filters mounted in series. SAS will also provide evacuated SUMMA canisters for use in collection of the particulate filter samples at the 241-AZ-702 stack. Sample preparation procedures are listed in Table 2-1.

Table 2-1. Sample Preparation Procedures

\begin{tabular}{|l|l|l|}
\hline Sample container & Organization & Preparation Procedure \\
\hline SUMMA canister & SAS & LO-080-406 \\
\hline TST & SAS & LA-549-403 \\
\hline STT & SAS & LA-549-402 \\
\hline PUF & SAS & N/A \\
\hline Particulate filter assembly & CPO, SAS & N/A \\
\hline
\end{tabular}

Note:

$\mathrm{N} / \mathrm{A}=$ not applicable

${ }^{1}$ SUMMA is a trademark of Tecktronics of Ohio. 


\subsection{SAMPLE COLLECTION}

CPO will be responsible for the collection of particulate filter samples at the 241-AZ-702 stack and for the collection of all SUMMA canister samples. SAS will be responsible for the collection of particulate filter samples at the 296-C-006 stack and for the collection of all TST, STT, and PUF samples.

CPO sampling activities will be performed in accordance with work package ES-99-0055 for the 296-C-006 stack and ambient air samples and work package ES-99-0054 for sampling at the 241-AZ-702 stack samples. SAS sampling activities will be performed in accordance with work package 2E-99-143 and procedure LO-080-400, Vapor Sampling Using the NonElectrical Vapor Sampling System (NEVS).

Tubing used in the collection of vapor samples must be stainless steel, Teflort ${ }^{2}$, or Tefloncoated and the length of the tubing used should be minimized. No C-flex or tygon tubing should be used upstream of the sample containers.

The samples shall include the following quality control (QC) samples: one TST, STT, and PUF ambient air field blank; and one TST, STT, and PUF trip blank. The trip and field blanks are to accompany the vapor samples to the laboratory. For specific information concerning sample and blank handling, custody, and transport, refer to the requirements in Section 4.0.

CPO and SAS shall record (or calculate) the following information for each sample collected: sample number, start and stop times for the collection of each sample, the TOC reading at the start of the collection of each sample, sample volumes, and any anomalous sampling conditions.

Particulate filter samples shall be shipped by CPO to the Waste Sampling and Characterization Facility (WSCF) for radiation screening analysis. The remaining samples shall be stored by $\mathrm{CPO}$ until radiation screening is completed and the samples can be released for analysis. Upon completion of the radiation screening and release of the samples, the samples shall be transferred to SAS for analysis.

Tables 2-2, 2-3, and 2-4 provide the sequence of sampling activities for 296-C-006 stack, ambient air, and 241-AZ-702 stack samples, respectively. The samples are summarized in Table 2-5. Unlike the sampling during Phase I of the process test, SUMMA, TST, STT, and PUF samples will not be collected through particulate filters. The particulate filter samples shall be collected separately, and the filter assembly removed from the sampling port prior to collecting the other samples.

\footnotetext{
${ }^{2}$ Teflon is a registered trademark of I. E. DuPont De Nemours and Company
} 
At a minimum, the following samples are required from the 296-C-006 stack:

- $\quad$ One set of samples before the pumps are started (baseline samples)

- $\quad$ One to three sets of samples after the pumps have started, as the stack TOC (as measured by the flame ionization detector [FID]) is increasing

- $\quad$ Two to three sets of samples during peak or steady state TOC concentrations

- $\quad$ One set of samples after the pumps are shut down and when TOC concentrations are subsiding.

To ensure that these samples are obtained, it is recommended that one set of samples be collected from the 296-C-006 stack once per hour (after pump start up) or when the TOC concentration has increased by 50 parts per million by volume (ppmv), whichever comes first (see Table 2-2). When three sets of samples have been collected at the peak/steady state TOC concentration, no further samples (except the set of post-shut-down samples) are required from the 296-C-006 stack. Sample collection or the suspension of sampling activities may also occur at the instruction of the test director. The sampling activities of SAS and CPO shall be coordinated so that, for each set of samples, the SUMMA canisters, TSTs, STTs, and PUFs are obtained at about the same time.

Ambient air samples in the $\mathrm{C}$ tank farm will consist of samples at three locations: 1) near the 296-C-006 stack, 2) near the fresh air truck, and 3) downwind of the stack at the fresh air boundary. Samples shall be obtained at these locations before the sluicing pumps are started and again when the TOC concentrations in the stack reach their peak or steady state concentrations.

Samples from the 241-AZ-702 stack will be obtained before the sluicing pumps are started and when the TOC concentration in the $241-\mathrm{AZ}-702$ stack has reached 25 ppmv and 50 ppmv. If 25 ppmv is not reached, two sets of samples shall be obtained when the TOC concentration in the 241-AZ-702 stack reached peak/steady state concentration. If the TOC concentration exceeds 25 ppmv but not 50 ppmv, one set of samples shall be obtained at 25 ppmv and the other shall be obtained when the TOC concentration in the 241-AZ-702 stack reached peak/steady state concentration.

Any decisions, observations, or deviations affecting this SAP shall be documented in controlled notebooks/or work packages and justified in the deliverable report. 
HNF-4030, Rev. 0

Table 2-2. List of Samples and Activities for 296-C-006 Stack (7 sheets).

\begin{tabular}{|c|c|c|c|c|}
\hline $\begin{array}{l}\text { Sample } \\
\text { Code }\end{array}$ & Sample/Activity Description & $\begin{array}{l}\text { Sampler Position } \\
\text { During Collection }\end{array}$ & $\begin{array}{l}\text { Sample } \\
\text { Volume }\end{array}$ & $\begin{array}{c}\text { Sample } \\
\text { Duration } \\
\text { or Flow } \\
\text { Rate }\end{array}$ \\
\hline--- & Perform leak test of NEVS & $\mathrm{N} / \mathrm{A}$ & N/A & N/A \\
\hline \multicolumn{5}{|c|}{ Baseline samples } \\
\hline 201 & $\begin{array}{l}\text { Collect particulate filter sample before pump } \\
\text { is started }\end{array}$ & 296-C-006 Stack & $1 \mathrm{~L}$ & $\begin{array}{l}\leq 500 \\
\mathrm{~mL} / \mathrm{min}\end{array}$ \\
\hline 202 & $\begin{array}{l}\text { Collect baseline SUMMA canister before } \\
\text { pump is started }\end{array}$ & 296-C-006 Stack & $6 \mathrm{~L}$ & $2 \mathrm{~min}$ \\
\hline 203 & Collect baseline TST before pump is started & 296-C-006 Stack & $0.5 \mathrm{~L}$ & $\begin{array}{l}\preceq 500 \\
\mathrm{~mL} / \mathrm{min}\end{array}$ \\
\hline 204 & Collect baseline STT before pump is started & 296-C-006 Stack & $6 \mathrm{~L}$ & $\begin{array}{l}\leq 500 \\
\mathrm{~mL} / \mathrm{min}\end{array}$ \\
\hline 205 & $\begin{array}{l}\text { Collect baseline PUF trap before pump is } \\
\text { started }\end{array}$ & 296-C-006 Stack & $25 \mathrm{~L}$ & $\unlhd 5 \mathrm{~L} / \mathrm{min}$ \\
\hline \multicolumn{5}{|c|}{ Sampling during sluicing operations } \\
\hline \multicolumn{5}{|c|}{ Collect sample set \#I one hr after pumps start or when TOC rises to 50 ppmy, whichever comes first. } \\
\hline 206 & Collect particulate filter sample & 296-C-006 Stack & $1 \mathrm{~L}$ & $\begin{array}{l}\leq 500 \\
\mathrm{~mL} / \mathrm{min}\end{array}$ \\
\hline 207 & Collect SUMMA canister & 296-C-006 Stack & $6 \mathrm{~L}$ & $2 \min$ \\
\hline 208 & Collect TST & 296-C-006 Stack & $0.5 \mathrm{~L}$ & $\begin{array}{l}\leq 500 \\
\mathrm{~mL} / \mathrm{min}\end{array}$ \\
\hline 209 & Collect STT & 296-C-006 Stack & $6 \mathrm{~L}$ & $\begin{array}{l}\leq 500 \\
\mathrm{~mL} / \mathrm{min}\end{array}$ \\
\hline 210 & Collect PUF trap & 296-C-006 Stack & $25 \mathrm{~L}$ & $\preceq 5 \mathrm{~L} / \mathrm{min}$ \\
\hline \multicolumn{5}{|c|}{$\begin{array}{l}\text { Collect sample set \#2 one hour after collection of sample set } \# 1 \text { or when TOC is } 50 \text { ppmy above the reading } \\
\text { when sample set \#1 was collected, whichever comes first }\end{array}$} \\
\hline $2 ! 1$ & Collect particulate filter sample & 296-C-006 Stack & $1 \mathrm{~L}$ & $\begin{array}{l}\leq 500 \\
\mathrm{~mL} / \mathrm{min}\end{array}$ \\
\hline 212 & Collect SUMMA canister & 296-C-006 Stack & $6 \mathrm{~L}$ & $2 \mathrm{~min}$ \\
\hline 213 & Collect TST & 296-C-006 Stack & $0.5 \mathrm{~L}$ & $\begin{array}{l}\leq 500 \\
\mathrm{~mL} / \mathrm{min}\end{array}$ \\
\hline 214 & Collect STT & 296-C-006 Stack & $6 \mathrm{~L}$ & $\begin{array}{l}\leq 500 \\
\mathrm{~mL} / \mathrm{min}\end{array}$ \\
\hline 215 & Collect PUF trap & 296-C-006 Stack & $25 \mathrm{~L}$ & $\lessgtr 5 \mathrm{~L} / \mathrm{min}$ \\
\hline
\end{tabular}


HNF-4030, Rev. 0

Table 2-2. List of Samples and Activities for 296-C-006 Stack (7 sheets).

\begin{tabular}{|c|c|c|c|c|}
\hline $\begin{array}{c}\text { Sample } \\
\text { Code }\end{array}$ & Sample/Activity Description & $\begin{array}{l}\text { Sampler Position } \\
\text { During Collection }\end{array}$ & $\begin{array}{l}\text { Sample } \\
\text { Volume }\end{array}$ & $\begin{array}{c}\text { Sample } \\
\text { Duration } \\
\text { or Flow } \\
\text { Rate }\end{array}$ \\
\hline \multicolumn{5}{|c|}{$\begin{array}{l}\text { Collect sample set } \# 3 \text { one hour after collection of sample set } \# 2 \text { or when } \mathrm{TOC} \text { is } 50 \mathrm{ppmy} \text { above the reading } \\
\text { when sample set } \# 2 \text { was collected, whichever comes first. }\end{array}$} \\
\hline 216 & Collect particulate filter sample & 296-C-006 Stack & $1 \mathrm{~L}$ & $\begin{array}{l}\leq 500 \\
\mathrm{~mL} / \mathrm{min}\end{array}$ \\
\hline 217 & Collect SUMMA canister & 296-C-006 Stack & $6 \mathrm{~L}$ & $2 \min$ \\
\hline 218 & Collect TST & 296-C-006 Stack & $0.5 \mathrm{~L}$ & $\begin{array}{l}\unlhd 500 \\
\mathrm{~mL} / \mathrm{min}\end{array}$ \\
\hline 219 & Collect STT & 296-C-006 Stack & $6 \mathrm{~L}$ & $\begin{array}{l}\leq 500 \\
\mathrm{~mL} / \mathrm{min}\end{array}$ \\
\hline 220 & Collect PUF trap & 296-C-006 Stack & $25 \mathrm{~L}$ & $\leq 5 \mathrm{~L} / \mathrm{min}$ \\
\hline \multicolumn{5}{|c|}{$\begin{array}{l}\text { Collect sample set } \# 4 \text { one hour after collection of sample set } \# 3 \text { or when TOC is } 50 \text { ppmv above the reading } \\
\text { when sample set } \# 3 \text { was collected, whichever comes first }\end{array}$} \\
\hline 221 & Collect particulate filter sample & 296-C-006 Stack & $1 \mathrm{~L}$ & $\begin{array}{l}\leq 500 \\
\mathrm{~mL} / \mathrm{min}\end{array}$ \\
\hline 222 & Collect SUMMA canister & 296-C-006 Stack & $6 \mathrm{~L}$ & $2 \mathrm{~min}$ \\
\hline 223 & Collect TST & 296-C-006 Stack & $0.5 \mathrm{~L}$ & $\begin{array}{l}\leq 500 \\
\mathrm{~mL} / \mathrm{min}\end{array}$ \\
\hline 224 & Collect STT & 296-C-006 Stack & $6 \mathrm{~L}$ & $\begin{array}{l}\leq 500 \\
\mathrm{~mL} / \mathrm{min}\end{array}$ \\
\hline 225 & Collect PUF trap & 296-C-006 Stack & $25 \mathrm{~L}$ & $\leq 5 \mathrm{~L} / \mathrm{min}$ \\
\hline \multicolumn{5}{|c|}{$\begin{array}{l}\text { Collect sample set \#5 one hour after collection of sample set } \# 4 \text { or when TOC is } 50 \text { ppmy above the reading } \\
\text { when sample set } \# 4 \text { was collected, whichever comes first }\end{array}$} \\
\hline 226 & Collect particulate filter sample & 296-C-006 Stack & $1 \mathrm{~L}$ & $\begin{array}{l}\unlhd 500 \\
\mathrm{~mL} / \mathrm{min}\end{array}$ \\
\hline 227 & Collect SUMMA canister & 296-C-006 Stack & $6 \mathrm{~L}$ & $2 \mathrm{~min}$ \\
\hline 228 & Collect TST & 296-C-006 Stack & $0.5 \mathrm{~L}$ & $\begin{array}{l}500 \\
\mathrm{~mL} / \mathrm{min}\end{array}$ \\
\hline 229 & Collect STT & 296-C-006 Stack & $6 \mathrm{~L}$ & $\begin{array}{l}₫ 500 \\
\mathrm{~mL} / \mathrm{min}\end{array}$ \\
\hline 230 & Collect PUF trap & 296-C-006 Stack & $25 \mathrm{~L}$ & $\leq 5 \mathrm{~L} / \mathrm{min}$ \\
\hline
\end{tabular}


HNF-4030, Rev. 0

Table 2-2. List of Samples and Activities for 296-C-006 Stack (7 sheets).

\begin{tabular}{|c|c|c|c|c|}
\hline $\begin{array}{l}\text { Sample } \\
\text { Code }\end{array}$ & Sample/Activity Description & $\begin{array}{l}\text { Sampler Position } \\
\text { During Collection }\end{array}$ & Sample & $\begin{array}{c}\text { Sample } \\
\text { Duration } \\
\text { or Flow } \\
\text { Rate }\end{array}$ \\
\hline \multicolumn{5}{|c|}{$\begin{array}{l}\text { Collect sample set \#6 one hour after collection of sample set } \# 5 \text { or when TOC is } 50 \text { ppmy above the reading } \\
\text { when sample set } \# 5 \text { was collected, whichever comes first. }\end{array}$} \\
\hline 231 & Collect particulate filter sample & 296-C-006 Stack & $1 \mathrm{~L}$ & $\begin{array}{l}\leq 500 \\
\mathrm{~mL} / \mathrm{min}\end{array}$ \\
\hline 232 & Collect SUMMA canister & 296-C-006 Stack & $6 \mathrm{~L}$ & $2 \mathrm{~min}$ \\
\hline 233 & Collect TST & 296-C-006 Stack & $0.5 \mathrm{~L}$ & $\begin{array}{l}\leq 500 \\
\mathrm{~mL} / \mathrm{min}\end{array}$ \\
\hline 234 & Collect STT & 296-C-006 Stack & $6 \mathrm{~L}$ & $\begin{array}{l}\leq 500 \\
\mathrm{~mL} / \mathrm{min}\end{array}$ \\
\hline 235 & Collect PUF trap & 296-C-006 Stack & $25 \mathrm{~L}$ & $\unlhd 5 \mathrm{~L} / \mathrm{min}$ \\
\hline \multicolumn{5}{|c|}{$\begin{array}{l}\text { Collect sample set \#7 one hour after collection of sample set } \$ 6 \text { or when roC is } 50 \text { ppmv above the reading } \\
\text { when sample set } \# 6 \text { was collected, whichever comes first }\end{array}$} \\
\hline 236 & Collect particulate filter sample & 296-C-006 Stack & $1 \mathrm{~L}$ & $\begin{array}{l}\leq 500 \\
\mathrm{~mL} / \mathrm{min}\end{array}$ \\
\hline 237 & Collect SUMMA canister & 296-C-006 Stack & $6 \mathrm{~L}$ & $2 \mathrm{~min}$ \\
\hline 238 & Collect TST & 296-C-006 Stack & $0.5 \mathrm{~L}$ & $\begin{array}{l}\leq 500 \\
\mathrm{~mL} / \mathrm{min}\end{array}$ \\
\hline 239 & Collect STT & 296-C-006 Stack & $6 \mathrm{~L}$ & $\begin{array}{l}\leq 500 \\
\mathrm{~mL} / \mathrm{min}\end{array}$ \\
\hline 240 & Collect PUF trap & 296-C-006 Stack & $25 \mathrm{~L}$ & $\preceq 5 \mathrm{~L} / \mathrm{min}$ \\
\hline \multicolumn{5}{|c|}{$\begin{array}{l}\text { Collect sample set } \# 8 \text { one hour after collection of sample set } \# 7 \text { or when } T O C \text { is } 50 \text { ppmy above the reading } \\
\text { when sample set } \# 7 \text { was collected, whichever comes first }\end{array}$} \\
\hline 241 & Collect particulate filter sample & 296-C-006 Stack & $1 \mathrm{~L}$ & $\begin{array}{l}\leq 500 \\
\mathrm{~mL} / \mathrm{min}\end{array}$ \\
\hline 242 & Collect SUMMA canister & 296-C-006 Stack & $6 \mathrm{~L}$ & $2 \mathrm{~min}$ \\
\hline 243 & Collect TST & 296-C-006 Stack & $0.5 \mathrm{~L}$ & $\begin{array}{l}\leq 500 \\
\mathrm{~mL} / \mathrm{min}\end{array}$ \\
\hline 244 & Collect STT & 296-C-006 Stack & $6 \mathrm{~L}$ & $\begin{array}{l}\leq 500 \\
\mathrm{~mL} / \mathrm{min}\end{array}$ \\
\hline 245 & Collect PUF trap & 296-C-006 Stack & $25 \mathrm{~L}$ & $\preceq 5 \mathrm{~L} / \mathrm{min}$ \\
\hline
\end{tabular}


HNF-4030, Rev. 0

Table 2-2. List of Samples and Activities for 296-C-006 Stack (7 sheets).

\begin{tabular}{|c|c|c|c|c|}
\hline $\begin{array}{l}\text { Sample } \\
\text { Code }\end{array}$ & Sample/Activity Description & $\begin{array}{l}\text { Sampler Position } \\
\text { During Collection }\end{array}$ & $\begin{array}{l}\text { Sample } \\
\text { Volume }\end{array}$ & $\begin{array}{c}\text { Sample } \\
\text { Duration } \\
\text { or Flow } \\
\text { Rate' }\end{array}$ \\
\hline \multicolumn{5}{|c|}{$\begin{array}{l}\text { Collect sample set } \# 9 \text { one hour after collection of sample set } \# 8 \text { or when TOC is } 50 \text { ppmv above the reading } \\
\text { when sample set } \# 8 \text { was collected, whichever comes first }\end{array}$} \\
\hline 246 & Collect particulate filter sample & 296-C-006 Stack & $1 \mathrm{~L}$ & $\begin{array}{l}\leq 500 \\
\mathrm{~mL} / \mathrm{min}\end{array}$ \\
\hline 247 & Collect SUMMA canister & 296-C-006 Stack & $6 \mathrm{~L}$ & $2 \min$ \\
\hline 248 & Collect TST & 296-C-006 Stack & $0.5 \mathrm{~L}$ & $\begin{array}{l}\leq 500 \\
\mathrm{~mL} / \mathrm{min}\end{array}$ \\
\hline 249 & Collect STT & 296-C-006 Stack & $6 \mathrm{~L}$ & $\begin{array}{l}\leq 500 \\
\mathrm{~mL} / \mathrm{min}\end{array}$ \\
\hline 250 & Collect PUF trap & 296-C-006 Stack & $25 \mathrm{~L}$ & $\leq 5 \mathrm{~L} / \mathrm{min}$ \\
\hline \multicolumn{5}{|c|}{$\begin{array}{l}\text { Collect sample set } \# 10 \text { one hour after collection of sample set } \# 9 \text { or when } T O C \text { is } 50 \mathrm{ppmy} \text { above the reading } \\
\text { when sample set } \# 9 \text { was collected, whichever comes first. }\end{array}$} \\
\hline 251 & Collect particulate filter sample & 296-C-006 Stack & $1 \mathrm{~L}$ & $\begin{array}{l}\leq 500 \\
\mathrm{~mL} / \mathrm{min}\end{array}$ \\
\hline 252 & Collect SUMMA canister & 296-C-006 Stack & $6 \mathrm{~L}$ & $2 \mathrm{~min}$ \\
\hline 253 & Collect TST & 296-C-006 Stack & $0.5 \mathrm{~L}$ & $\begin{array}{l}\leq 500 \\
\mathrm{~mL} / \mathrm{min}\end{array}$ \\
\hline 254 & Collect STT & 296-C-006 Stack & $6 \mathrm{~L}$ & $\begin{array}{l}\leq 500 \\
\mathrm{~mL} / \mathrm{min}\end{array}$ \\
\hline 255 & Collect PUF trap & 296-C-006 Stack & $25 \mathrm{~L}$ & $₫ 5 \mathrm{~L} / \mathrm{min}$ \\
\hline \multicolumn{5}{|c|}{$\begin{array}{l}\text { Collect sample set \#11 one hour after collection of sample set } \# 10 \text { or when } T O C \text { is } 50 \text { ppmv above the reading } \\
\text { when sample set } \# 10 \text { was collected, whichever comes first }\end{array}$} \\
\hline 256 & Collect particulate filter sample & 296-C-006 Stack & $1 \mathrm{~L}$ & $\begin{array}{l}\leq 500 \\
\mathrm{~mL} / \mathrm{min}\end{array}$ \\
\hline 257 & Collect SUMMA canister & 296-C-006 Stack & $6 \mathrm{~L}$ & $2 \mathrm{~min}$ \\
\hline 258 & Collect TST & 296-C-006 Stack & $0.5 \mathrm{~L}$ & $\begin{array}{l}\leq 500 \\
\mathrm{~mL} / \mathrm{min}\end{array}$ \\
\hline 259 & Collect STT & 296-C-006 Stack & $6 \mathrm{~L}$ & $\begin{array}{l}\leq 500 \\
\mathrm{~mL} / \mathrm{min}\end{array}$ \\
\hline 260 & Collect PUF trap & 296-C-006 Stack & $25 \mathrm{~L}$ & $\leq 5 \mathrm{~L} / \mathrm{min}$ \\
\hline
\end{tabular}


HNF-4030, Rev. 0

Table 2-2. List of Samples and Activities for 296-C-006 Stack (7 sheets).

\begin{tabular}{|c|c|c|c|c|}
\hline $\begin{array}{l}\text { Sample } \\
\text { Code }\end{array}$ & Sample/Activity Description & $\begin{array}{l}\text { Sampler Position } \\
\text { During Collection }\end{array}$ & $\begin{array}{l}\text { Sample } \\
\text { Volume }\end{array}$ & $\begin{array}{c}\text { Sample } \\
\text { Duration } \\
\text { or Flow } \\
\text { Rate }^{1}\end{array}$ \\
\hline \multicolumn{5}{|c|}{$\begin{array}{l}\text { Collect sample set } \# 12 \text { one hour after collection of sample set } \# 11 \text { or when TOC is } 50 \text { ppmy above the reading } \\
\text { when sample set } \# 11 \text { was collected, whichever comes first }\end{array}$} \\
\hline 261 & Collect particulate filter sample & 296-C-006 Stack & $1 \mathrm{~L}$ & $\begin{array}{l}\leq 500 \\
\mathrm{~mL} / \mathrm{min}\end{array}$ \\
\hline 262 & Collect SUMMA canister & 296-C-006 Stack & $6 \mathrm{~L}$ & $2 \mathrm{~min}$ \\
\hline 263 & Collect TST & 296-C-006 Stack & $0.5 \mathrm{~L}$ & $\begin{array}{l}\leq 500 \\
\mathrm{~mL} / \mathrm{min}\end{array}$ \\
\hline 264 & Collect STT & 296-C-006 Stack & $6 \mathrm{~L}$ & $\begin{array}{l}\preceq 500 \\
\mathrm{~mL} / \mathrm{min}\end{array}$ \\
\hline 265 & Collect PUF trap & 296-C-006 Stack & $25 \mathrm{~L}$ & $\leq 5 \mathrm{~L} / \mathrm{min}$ \\
\hline \multicolumn{5}{|c|}{$\begin{array}{l}\text { Collect sample set \#13 one hour after collection of sample set } \# 12 \text { or when TOC is } 50 \text { ppmv above the reading } \\
\text { when sample set } \# 12 \text { was collected, whichever comes first. }\end{array}$} \\
\hline 266 & Collect particulate filter sample & 296-C-006 Stack & $1 \mathrm{~L}$ & $\begin{array}{l}\checkmark 500 \\
\mathrm{~mL} / \mathrm{min}\end{array}$ \\
\hline 267 & Collect SUMMA canister & 296-C-006 Stack & $6 \mathrm{~L}$ & $2 \min$ \\
\hline 268 & Collect TST & 296-C-006 Stack & $0.5 \mathrm{~L}$ & $\begin{array}{l}\leq 500 \\
\mathrm{~mL} / \mathrm{min}\end{array}$ \\
\hline 269 & Collect STT & 296-C-006 Stack & $6 \mathrm{~L}$ & $\begin{array}{l}\leq 500 \\
\mathrm{~mL} / \mathrm{min}\end{array}$ \\
\hline 270 & Collect PUF trap & 296-C-006 Stack & $25 \mathrm{~L}$ & $\leq 5 \mathrm{~L} / \mathrm{min}$ \\
\hline \multicolumn{5}{|c|}{$\begin{array}{l}\text { Collect sample set } \# 14 \text { one hour after collection of sample set } \# 13 \text { or when TOC is } 50 \text { ppmv above the reading } \\
\text { when sample set } \# 13 \text { was collected, whichever comes first }\end{array}$} \\
\hline 271 & Collect particulate filter sample & 296-C-006 Stack & $1 \mathrm{~L}$ & $\begin{array}{l}\leq 500 \\
\mathrm{~mL} / \mathrm{min}\end{array}$ \\
\hline 272 & Collect SUMMA canister & 296-C-006 Stack & $6 \mathrm{~L}$ & $5 \mathrm{~min}$ \\
\hline 273 & Collect TST & 296-C-006 Stack & $0.5 \mathrm{~L}$ & $\begin{array}{l}\leq 500 \\
\mathrm{~mL} / \mathrm{min}\end{array}$ \\
\hline 274 & Collect STT & 296-C-006 Stack & $6 \mathrm{~L}$ & $\begin{array}{l}\leq 500 \\
\mathrm{~mL} / \mathrm{min}\end{array}$ \\
\hline 275 & Collect PUF trap & 296-C-006 Stack & $25 \mathrm{~L}$ & $\leq 5 \mathrm{~L} / \mathrm{min}$ \\
\hline
\end{tabular}


HNF-4030, Rev. 0

Table 2-2. List of Samples and Activities for 296-C-006 Stack (7 sheets).

\begin{tabular}{|c|c|c|c|c|}
\hline $\begin{array}{l}\text { Sample } \\
\text { Code }\end{array}$ & $\begin{array}{l}\text { Sample/Activity Description } \\
\qquad\end{array}$ & $\begin{array}{l}\text { Samplet Position } \\
\text { During Collection }\end{array}$ & $\begin{array}{l}\text { Sample } \\
\text { Volume }\end{array}$ & $\begin{array}{l}\text { Sample } \\
\text { Duration } \\
\text { or Flow } \\
\text { Ratel }\end{array}$ \\
\hline \multicolumn{5}{|c|}{$\begin{array}{l}\text { Collect sample set } \# 15 \text { one hour after collection of sample set } \# 14 \text { or when TOC is } 50 \text { ppmvabove the reading } \\
\text { when sample set } \# 14 \text { was collected, whichever comes first }\end{array}$} \\
\hline 276 & Collect particulate filter sample & 296-C-006 Stack & $1 \mathrm{~L}$ & $\begin{array}{l}\leq 500 \\
\mathrm{~mL} / \mathrm{min}\end{array}$ \\
\hline 277 & Collect SUMMA canister & 296-C-006 Stack & $6 \mathrm{~L}$ & $2 \mathrm{~min}$ \\
\hline 278 & Collect TST & 296-C-006 Stack & $0.5 \mathrm{~L}$ & $\begin{array}{l}\leq 500 \\
\mathrm{~mL} / \mathrm{min}\end{array}$ \\
\hline 279 & Collect STT & 296-C-006 Stack & $6 \mathrm{~L}$ & $\begin{array}{l}\preceq 500 \\
\mathrm{~mL} / \mathrm{min}\end{array}$ \\
\hline 280 & Collect PUF trap & 296-C-006 Stack & $25 \mathrm{~L}$ & $\leq 5 \mathrm{~L} / \mathrm{min}$ \\
\hline \multicolumn{5}{|c|}{$\begin{array}{c}\text { Collect sample set \#16 one hour after collection of sample set } \# 15 \text { or when TOC is } 50 \text { ppmy above the reading } \\
\text { when sample set } \# 15 \text { was collected, whichever comes first }\end{array}$} \\
\hline 281 & Collect particulate filter sample & 296-C-006 Stack & $1 \mathrm{~L}$ & $\begin{array}{l}\leq 500 \\
\mathrm{~mL} / \mathrm{min}\end{array}$ \\
\hline 282 & Collect SUMMA canister & 296-C-006 Stack & $6 \mathrm{~L}$ & $2 \mathrm{~min}$ \\
\hline 283 & Collect TST & 296-C-006 Stack & $0.5 \mathrm{~L}$ & $\begin{array}{l}\leq 500 \\
\mathrm{~mL} / \mathrm{min}\end{array}$ \\
\hline 284 & Collect STT & 296-C-006 Stack & $6 \mathrm{~L}$ & $\begin{array}{l}\leq 500 \\
\mathrm{~mL} / \mathrm{min}\end{array}$ \\
\hline 285 & Collect PUF trap & 296-C-006 Stack & $25 \mathrm{~L}$ & $\leq 5 \mathrm{~L} / \mathrm{min}$ \\
\hline \multicolumn{5}{|c|}{$\begin{array}{l}\text { Collect sample set \#17 one hour after collection of sample set } \# 16 \text { or when } T O C \text { is } 50 \text { ppmv above the reading } \\
\text { when sample set } \# 16 \text { was collected, whichever comes first }\end{array}$} \\
\hline 286 & Collect particulate filter sample & 296-C-006 Stack & $1 \mathrm{~L}$ & $\begin{array}{l}\leq 500 \\
\mathrm{~mL} / \mathrm{min}\end{array}$ \\
\hline 287 & Collect SUMMA canister & 296-C-006 Stack & $6 \mathrm{~L}$ & $2 \mathrm{~min}$ \\
\hline 288 & Collect TST & 296-C-006 Stack & $0.5 \mathrm{~L}$ & $\begin{array}{l}\leq 500 \\
\mathrm{~mL} / \mathrm{min}\end{array}$ \\
\hline 289 & Collect STT & 296-C-006 Stack & $6 \mathrm{~L}$ & $\begin{array}{l}\leq 500 \\
\mathrm{~mL} / \mathrm{min}\end{array}$ \\
\hline 290 & Collect PUF trap & 296-C-006 Stack & $25 \mathrm{~L}$ & $\leq 5 \mathrm{~L} / \mathrm{min}$ \\
\hline
\end{tabular}


HNF-4030, Rev. 0

Table 2-2. List of Samples and Activities for 296-C-006 Stack (7 sheets).

\begin{tabular}{|c|c|c|c|c|}
\hline $\begin{array}{l}\text { Sample } \\
\text { Code }\end{array}$ & Sample/Activity Description & $\begin{array}{l}\text { Sampler Position } \\
\text { During Collection }\end{array}$ & $\begin{array}{l}\text { Sample } \\
\text { Volume }\end{array}$ & $\begin{array}{l}\text { Sample } \\
\text { Duration } \\
\text { or Flow } \\
\text { Rate }\end{array}$ \\
\hline \multicolumn{5}{|c|}{ Post shut down samples } \\
\hline 291 & $\begin{array}{l}\text { Collect post shut-down particulate filter } \\
\text { sample }\end{array}$ & 296-C-006 Stack & $1 \mathrm{~L}$ & $\begin{array}{l}₫ 500 \\
\mathrm{~mL} / \mathrm{min}\end{array}$ \\
\hline 292 & Collect post shut-down SUMMA canister & 296-C-006 Stack & $6 \mathrm{~L}$ & $2 \mathrm{~min}$ \\
\hline 293 & Collect post shut-down TST & 296-C-006 Stack & $0.5 \mathrm{~L}$ & $\begin{array}{l}\preceq 500 \\
\mathrm{~mL} / \mathrm{min}\end{array}$ \\
\hline 294 & Collect post shut-down STT & 296-C-006 Stack & $6 \mathrm{~L}$ & $\begin{array}{l}\leq 500 \\
\mathrm{~mL} / \mathrm{min}\end{array}$ \\
\hline 295 & Collect post shut-down PUF trap & 296-C-006 Stack & $25 \mathrm{~L}$ & $\leq 5 \mathrm{~L} / \mathrm{min}$ \\
\hline 296 & Collect final particulate filter sample & 296-C-006 Stack & $1 \mathrm{~L}$ & $\begin{array}{l}\leq 500 \\
\mathrm{~mL} / \mathrm{min}\end{array}$ \\
\hline \multicolumn{5}{|c|}{ Blanks } \\
\hline 297 & Store TST Trip Blank & -- & --- & $\cdots$ \\
\hline 298 & Store STT Trip Blank & --- & --- & -.. \\
\hline 299 & Store PUF Trip Blank & --- & -- & --- \\
\hline 300 & Open, close, and store TST Field Blank & --- & $-\cdots$ & --- \\
\hline 301 & Open, close, and store STT Field Blank & -- & $\cdots$ & --- \\
\hline 302 & Open, close, and store PUF Field Blank & -- & $\cdots$ & --- \\
\hline
\end{tabular}

Note:

${ }^{1} \mathrm{CPO}$ and SAS may adjust sample durations and flow rates as necessary to collect the samples. 
HNF-4030, Rev. 0

Table 2-3. List of Samples and Activities for Ambient Air Sampling in C Tank Farm

\begin{tabular}{|c|c|c|c|c|}
\hline $\begin{array}{l}\text { Sample } \\
\text { Code }\end{array}$ & Sample/Activity Description & $\begin{array}{l}\text { Sampler Position } \\
\text { During Collection }\end{array}$ & $\begin{array}{l}\text { Sample } \\
\text { Volume: }\end{array}$ & $\begin{array}{l}\text { Sample } \\
\text { Duration } \\
\text { or Rate }\end{array}$ \\
\hline \multicolumn{5}{|c|}{ Baseline samples } \\
\hline 201 & $\begin{array}{l}\text { Collect baseline SUMMA before pump is } \\
\text { started. }\end{array}$ & near 296-C-006 stack & $6 \mathrm{~L}$ & $1 \mathrm{~min}$ \\
\hline 202 & Collect baseline TST before pump is started & near $296-C-006$ stack & $0.5 \mathrm{~L}$ & $\begin{array}{l}\leq 500 \\
\mathrm{~mL} / \mathrm{min}\end{array}$ \\
\hline 203 & Collect baseline STT before pump is started & near $296-C-006$ stack & $6 \mathrm{~L}$ & $\begin{array}{l}\leq 500 \\
\mathrm{~mL} / \mathrm{min}\end{array}$ \\
\hline 204 & Collect baseline PUF before pump is started & near 296-C-006 stack & $25 \mathrm{~L}$ & $\preceq 5 \mathrm{~L} / \mathrm{min}$ \\
\hline 205 & $\begin{array}{l}\text { Collect baseline SUMMA before pump is } \\
\text { started. }\end{array}$ & near fresh air truck & $6 \mathrm{~L}$ & $1 \mathrm{~min}$ \\
\hline 206 & $\begin{array}{l}\text { Collect baseline SUMMA before pump is } \\
\text { started }\end{array}$ & $\begin{array}{l}\text { downwind at the } \\
\text { fresh air boundary }\end{array}$ & $6 \mathrm{~L}$ & $1 \mathrm{~min}$ \\
\hline \multicolumn{5}{|c|}{ Samples at peak/steady state TOC concentrations } \\
\hline 207 & $\begin{array}{l}\text { Collect SUMMA when stack TOC } \\
\text { concentration has reached peak/steady state }\end{array}$ & near 296-C-006 stack & $6 \mathrm{~L}$ & $1 \mathrm{~min}$ \\
\hline 208 & $\begin{array}{l}\text { Collect TST when stack TOC concentration } \\
\text { has reached peak/steady state }\end{array}$ & near 296-C-006 stack & $0.5 \mathrm{~L}$ & $\begin{array}{l}\mathrm{mL} / \mathrm{min}\end{array}$ \\
\hline 209 & $\begin{array}{l}\text { Collect STT when stack TOC concentration } \\
\text { has reached peak/steady state }\end{array}$ & near 296-C-006 stack & $6 \mathrm{~L}$ & $\begin{array}{l}\leq 500 \\
\mathrm{~mL} / \mathrm{min}\end{array}$ \\
\hline 210 & $\begin{array}{l}\text { Collect PUF when stack TOC concentration } \\
\text { has reached peak/steady state }\end{array}$ & near 296-C-006 stack & $25 \mathrm{~L}$ & $\leq 5 \mathrm{~L} / \mathrm{min}$ \\
\hline 211 & $\begin{array}{l}\text { Collect SUMMA when stack TOC } \\
\text { concentration has reached peak/steady state }\end{array}$ & near fresh air truck & $6 \mathrm{~L}$ & $1 \mathrm{~min}$ \\
\hline 212 & $\begin{array}{l}\text { Collect SUMMA when stack TOC } \\
\text { concentration has reached peak/steady state }\end{array}$ & $\begin{array}{l}\text { downwind at the } \\
\text { fresh air boundary }\end{array}$ & $6 \mathrm{~L}$ & $1 \mathrm{~min}$ \\
\hline
\end{tabular}

Note:

'CPO and SAS may adjust sample durations and flow rates as necessary to collect the samples. 
HNF-4030, Rev. 0

Table 2-4. List of Samples and Activities for 241-AZ-702 Stack

\begin{tabular}{|c|c|c|c|c|}
\hline $\begin{array}{l}\text { Sample } \\
\text { Code }\end{array}$ & Sample/Activity Description & $\begin{array}{l}\text { Sampler Position } \\
\text { During Collection }\end{array}$ & $\begin{array}{l}\text { Sample } \\
\text { Volume }\end{array}$ & $\begin{array}{l}\text { Sample } \\
\text { Duration } \\
\text { or Rate }\end{array}$ \\
\hline \multicolumn{5}{|c|}{ Baseline samples } \\
\hline 201 & Collect baseline particulate filter sample & 241-AZ-702 Stack & $6 \mathrm{~L}$ & $2 \mathrm{~min}$ \\
\hline 202 & Collect baseline SAS SUMMA & 241-AZ-702 Stack & $6 \mathrm{~L}$ & $2 \mathrm{~min}$ \\
\hline \multicolumn{5}{|c|}{ Sample during pump operation } \\
\hline 203 & $\begin{array}{l}\text { Collect particulate filter sample when stack } \\
\text { FID/PID reading is } 25 \mathrm{ppmv} \text { (or at } \\
\text { peak/steady state if } 25 \mathrm{ppmv} \text { is not reached) }\end{array}$ & 241-AZ-702 Stack & $6 \mathrm{~L}$ & $2 \mathrm{~min}$ \\
\hline 204 & $\begin{array}{l}\text { Collect SAS SUMMA when stack reading is } \\
25 \mathrm{ppmv} \text { (or at peak/steady state if } 25 \mathrm{ppmv} \\
\text { is not reached) }\end{array}$ & 241-AZ-702 Stack & $6 \mathrm{~L}$ & $2 \mathrm{~min}$ \\
\hline 205 & $\begin{array}{l}\text { Collect particulate filter sample when stack } \\
\text { FID/PID reading is } 50 \mathrm{ppmv} \text { (or at } \\
\text { peak/steady state if } 50 \mathrm{ppmv} \text { is not reached) }\end{array}$ & 241-AZ-702 Stack & $6 \mathrm{~L}$ & $2 \mathrm{~min}$ \\
\hline 206 & $\begin{array}{l}\text { Collect SAS SUMMA when stack reading is } \\
50 \mathrm{ppmv} \text { (or at peak/steady state if } 50 \mathrm{ppmv} \\
\text { is not reached) }\end{array}$ & 241-AZ-702 Stack & $6 \mathrm{~L}$ & $2 \mathrm{~min}$ \\
\hline \multicolumn{5}{|c|}{ After completion of sampling at $241-A Z-702$ stack } \\
\hline 207 & Collect final particulate fitter sample & 241-AZ-702 Stack & $6 \mathrm{~L}$ & $2 \mathrm{~min}$ \\
\hline
\end{tabular}

Note:

${ }^{1} \mathrm{CPO}$ and SAS may adjust sample durations and flow rates as necessary to collect the samples. 
HNF-4030, Rev. 0

Table 2-5. Vapor Sampling Summary (2 sheets).

\begin{tabular}{|c|c|c|c|c|}
\hline Sample location & Sample time & $\begin{array}{l}\text { Sample } \\
\text { type }\end{array}$ & Sample container & $\begin{array}{l}\text { Number of } \\
\text { samples }\end{array}$ \\
\hline \multirow[t]{3}{*}{--} & \multirow[t]{3}{*}{ Baseline } & \multirow[t]{3}{*}{ Trip blank } & TST & 1 \\
\hline & & & STT & 1 \\
\hline & & & PUF & 1 \\
\hline \multirow[t]{3}{*}{---} & \multirow[t]{3}{*}{ Baseline } & \multirow[t]{3}{*}{ Field blank } & TST & 1 \\
\hline & & & STT & 1 \\
\hline & & & PUF & 1 \\
\hline \multirow[t]{15}{*}{ 296-C-006 stack } & \multirow[t]{5}{*}{ Baseline } & \multirow{5}{*}{$\begin{array}{l}\text { Stack } \\
\text { vapor }\end{array}$} & SUMMA & 1 \\
\hline & & & TST & 1 \\
\hline & & & STT & 1 \\
\hline & & & PUF & 1 \\
\hline & & & Particulate filter assembly & 1 \\
\hline & \multirow[t]{5}{*}{ During sluicing } & \multirow{5}{*}{$\begin{array}{l}\text { Stack } \\
\text { vapor }\end{array}$} & SUMMA & up to 17 \\
\hline & & & TST & up to 17 \\
\hline & & & STT & up to 17 \\
\hline & & & PUF & up to 17 \\
\hline & & & Particulate filter assembly & up to 17 \\
\hline & \multirow[t]{5}{*}{ Post shut-down } & \multirow{5}{*}{$\begin{array}{l}\text { Stack } \\
\text { vapor }\end{array}$} & SUMMA & 1 \\
\hline & & & TST & 1 \\
\hline & & & STT & 1 \\
\hline & & & PUF & 1 \\
\hline & & & Particulate filter assembly & 2 \\
\hline \multirow{4}{*}{$\begin{array}{l}\text { Breathing Area, } \\
\text { C Tank Farm }\end{array}$} & \multirow[t]{4}{*}{ Baseline } & \multirow{4}{*}{$\begin{array}{l}\text { Ambient } \\
\text { air }\end{array}$} & SUMMA & 3 \\
\hline & & & TST & 1 \\
\hline & & & STT & 1 \\
\hline & & & PUF & 1 \\
\hline
\end{tabular}


HNF-4030, Rev. 0

Table 2-5. Vapor Sampling Summary (2 sheets).

\begin{tabular}{|c|c|c|c|c|}
\hline Sample location & Sample time & $\begin{array}{l}\text { Sample } \\
\text { type }\end{array}$ & Sample container & $\begin{array}{l}\text { Number of } \\
\text { samples }\end{array}$ \\
\hline \multirow{4}{*}{$\begin{array}{l}\text { Breathing Area, } \\
\text { C Tank Farm, } \\
\text { (continued) }\end{array}$} & \multirow[t]{4}{*}{ During sluicing } & \multirow{4}{*}{$\begin{array}{l}\text { Ambient } \\
\text { air }\end{array}$} & SUMMA & 3 \\
\hline & & & TST & 1 \\
\hline & & & STT & 1 \\
\hline & & & PUF & 1 \\
\hline \multirow{4}{*}{$\begin{array}{l}\text { 241-AZ-702 } \\
\text { stack }\end{array}$} & \multirow[t]{2}{*}{ Baseline } & \multirow{2}{*}{$\begin{array}{l}\text { Stack } \\
\text { vapor }\end{array}$} & SUMMA & 1 \\
\hline & & & Particulate filter assembly & 1 \\
\hline & \multirow[t]{2}{*}{ During sluicing } & \multirow{2}{*}{$\begin{array}{l}\text { Stack } \\
\text { vapor }\end{array}$} & SUMMA & 2 \\
\hline & & & Particulate filter assembly & 3 \\
\hline
\end{tabular}

Notes:

baseline $=$ prior to pump startup

PUF $=$ polyurethane foam filter

STT $=$ sorbent tube train

TST $=$ triple sorbent tube

Each particulate filter assembly contains two particulate filters mounted in series.

\subsection{RADIATION SCREENING}

Surveys using particulate filter samples from the 296-C-006 and the 241-AZ-702 ventilation systems will be used periodically during the process test to allow the samples to obtain a radiological release and ensure that the samples meet the SAS laboratory acceptance criteria. The particulate filter samples will be collected by CPO and SAS and sent to WSCF for analysis of both the upstream and downstream particulate filters in each particulate filter assembly. Analytical procedures are specified in Section 3.0. If the limits specified in Section 3.0 are exceeded the survey samples will be recounted every few days until the activity drops below the limits, allowing release of the samples. The results from the radiation screening shall be submitted to SAS and the Process Engineering point of contact for vapor sampling for evaluation. Tritium trap samples were collected and analyzed during phase I of the process test in December 1998. The results of these earlier tritium trap samples are adequate to release the phase II process test samples with regard to potential tritium contamination. Therefore, no tritium trap samples will be obtained during phase II of the process test. 
HNF-4030, Rev. 0

\subsection{LABORATORY ANALYSIS REQUIREMENTS}

The responsibilities of the analytical laboratories are given in this section. Additional quality control and deliverable requirements are given in Sections 4.0 and 7.0.

Vapor samples shall be analyzed by SAS in accordance with Table 3-1. Sorbent tube trains shall be analyzed for ammonia and oxides of nitrogen (nitric oxide and nitrogen dioxide). SUMMA canisters shall be analyzed for total non-methane hydrocarbons, hydrogen, nitrous oxide, methane, carbon monoxide, carbon dioxide, and selected organic analytes. TSTs shall be analyzed for selected organic analytes. PUF samples shall be analyzed for tri-butyl phosphate (TBP) and di-butyl phosphate (DBP).

Required analytes are those listed in Tables 3-2, 3-3, and 3-4. Quantitation limit goals for the Class A and B toxic air pollutants in these tables are documented in Mulkey (1995). In addition to the compounds listed, a determination is to be made for all other peaks that are at least 10 percent of the nearest internal standard. If possible, peaks smaller than 10 percent of the nearest internal standard should also be identified. All major constituents in the sample should be identified.

It is expected that not all samples will be analyzed. At a minimum, the laboratory shall analyze all trip blanks, field blanks, breathing area samples, baseline samples, post-shut down samples, at least two sets of samples from 296-C-006 stack, and at least one set of samples from the 241-AZ-702 stack. Prior to the receipt of the samples at the laboratory, the Process Engineering point of contact for vapor sampling shall provide SAS with a list identifying the stack samples to be analyzed.

Particulate filter samples shall be analyzed at the WSCF as discussed in Section 2.3 and in accordance with Table 3-1.

If any requested analyses cannot be performed, the Process Engineering point of contact for vapor sampling shall be notified. 
HNF-4030, Rev. 0

Table 3-1. Chemical and Radiological Analytical Requirements

\begin{tabular}{|c|c|c|c|c|c|c|c|c|c|c|}
\hline \multicolumn{11}{|c|}{ YAPOR ANAIYSES } \\
\hline \multirow{2}{*}{\multicolumn{2}{|c|}{$\begin{array}{l}\text { Proiect Name } \\
\text { Plan Number }\end{array}$}} & \multicolumn{3}{|l|}{ WRRS } & \multicolumn{3}{|c|}{ Comments } & \multicolumn{3}{|c|}{ Reporting Formats } \\
\hline & & \multirow{2}{*}{\multicolumn{3}{|c|}{ HNE-4030_ReV 0}} & \multirow{6}{*}{\multicolumn{3}{|c|}{$\begin{array}{l}\text { Field Blank - Required } \\
\text { Trip Blank - Required }\end{array}$}} & \multirow{2}{*}{$\begin{array}{l}\text { Format I } \\
\text { Eormat II }\end{array}$} & \multirow{2}{*}{\multicolumn{2}{|c|}{ Immediate Notification }} \\
\hline \multicolumn{2}{|l|}{ (2) } & & & & & & & & & \\
\hline \multicolumn{2}{|c|}{ Program Contact: } & \multicolumn{3}{|l|}{ J. W. Lentsch } & & & & Format III & \multicolumn{2}{|l|}{ Safety Screen } \\
\hline \multicolumn{2}{|c|}{ TWRS Contact: } & \multicolumn{3}{|l|}{ L. M. Sasaki } & & & & Format IV & \multicolumn{2}{|c|}{ Waste Management } \\
\hline \multicolumn{2}{|c|}{ Lab Contact (SAS): } & \multicolumn{3}{|l|}{ R. S. Viswanath } & & & & Format V & \multicolumn{2}{|c|}{ RCRA Compliance } \\
\hline & & & & & & & & Format VI & Special & \\
\hline \multicolumn{7}{|c|}{ PRIMARY ANALYSES } & \multicolumn{3}{|c|}{ CRITERIA } & \multirow{2}{*}{$\begin{array}{l}\text { REPORT } \\
\text { FORMAT }\end{array}$} \\
\hline $\begin{array}{l}\text { ANALYSIS } \\
\text { METHOD } \\
\end{array}$ & $\begin{array}{l}\text { PRIMARY } \\
\text { ANALYTE } \\
\end{array}$ & PROCEDURE & LAB & $\begin{array}{l}\text { SAMPLE } \\
\text { PREP }\end{array}$ & $\begin{array}{c}\text { SAMPLE } \\
\text { CONTAINER }\end{array}$ & $\begin{array}{l}\text { SURROGATE } \\
\text { SPIKE }\end{array}$ & $\begin{array}{c}\text { NOTIFICATION } \\
\text { LIMIT }\end{array}$ & PRECISION & ACCURACY & \\
\hline GC/MS & $\begin{array}{l}\text { Organic } \\
\text { Speciation }\end{array}$ & LA-523-404 & SAS & Direct & SUMMA & none & $\begin{array}{l}\geq 20 \% \text { LFL } \\
\text { and/or } 50 \% \text { IDLH }\end{array}$ & $\pm 25 \%$ & $70-130 \%$ & I, VI \\
\hline $\mathrm{GC} / \mathrm{TCD}$ & $\begin{array}{l}\mathrm{CO}_{2}, \mathrm{CO}, \\
\mathrm{CH}_{4}, \mathrm{H}_{2} \text {, and } \\
\mathrm{N}_{2} \mathrm{O}\end{array}$ & LA-523-409 & SAS & Direct & SUMMA & none & $\begin{array}{l}\geq 20 \% \text { LFL } \\
\text { and/or } 50 \% \text { IDLH }\end{array}$ & $\pm 25 \%$ & $70-130 \%$ & I, VI \\
\hline $\mathrm{GC} / \mathrm{FID}$ & $\begin{array}{l}\text { Total } \\
\text { nonmethane } \\
\text { hydrocarbons }\end{array}$ & LA-523-407 & SAS & Direct & SUMMA & none & $220 \% \mathrm{LFL}$ & $\pm 25 \%$ & $70-130 \%$ & I, VI \\
\hline IC & $\overline{\mathrm{NH}_{3}}$ & LA-533-402 & SAS & $\begin{array}{c}\mathrm{H}_{2} \mathrm{O} \\
\text { Extraction }\end{array}$ & STT & none & $\geq 150 \mathrm{ppmv}$ & $\pm 25 \%$ & $70-130 \%$ & I, VI \\
\hline IC & $\begin{array}{l}\text { Oxides of } \\
\text { nitrogen }\end{array}$ & LA-533-400 & SAS & $\begin{array}{c}\mathrm{H}_{2} \mathrm{O} \\
\text { Extraction }\end{array}$ & STT & none & $\geq 150 \mathrm{ppmv}$ & $\pm 25 \%$ & $70-130 \%$ & I, VI \\
\hline GC/MS & $\begin{array}{l}\text { Organic } \\
\text { Speciation }\end{array}$ & LA-523-408 & SAS & $\begin{array}{c}\text { Thermal } \\
\text { Desorption }\end{array}$ & TST & all & $\begin{array}{l}220 \% \text { LFL } \\
\text { and } / \text { or } 50 \% \text { IDLH }\end{array}$ & $\pm 25 \%$ & $70-130 \%$ & I, VI \\
\hline $\mathrm{GC} / \mathrm{MS}$ & TBP, DBP & LA- $523-428$ & SAS & $\begin{array}{c}\text { Solvent } \\
\text { Extraction }\end{array}$ & PUF & none & $\begin{array}{l}\geq 20 \% \text { LFL } \\
\text { and/or } 50 \% \text { IDLH }\end{array}$ & $\pm 25 \%$ & $70-130 \%$ & I, VI \\
\hline $\begin{array}{l}\text { Total } \alpha \text {, } \\
\text { total } B \text {, } \\
\gamma \text { Energy }\end{array}$ & Radionuclides & $\begin{array}{l}\text { LA-548-421 } \\
\text { LA-508-415 } \\
\text { LA-508-462 }\end{array}$ & WSCF & Direct & $\begin{array}{l}\text { Particulate } \\
\text { Filter }\end{array}$ & N/A & N/A & $\pm 25 \%$ & $70-130 \%$ & II \\
\hline
\end{tabular}

Notes:

$\mathrm{N} / \mathrm{A}=$ not applicable 
HNF-4030, Rev. 0

Table 3-2. Required Analytes: Regulatory

\begin{tabular}{|l|r|r|r|r|r|}
\hline \multicolumn{1}{|c|}{ Analyte } & $\begin{array}{r}\text { CAS } \\
\text { Number }\end{array}$ & $\begin{array}{r}\text { LFL } \\
\text { (ppmv) }\end{array}$ & $\begin{array}{r}\text { LFL } \\
\text { (ppmv) }\end{array}$ & $\begin{array}{r}50 \% \\
\text { IDLH } \\
\text { (ppmv) }\end{array}$ & $\begin{array}{r}\text { IDLH } \\
\text { (ppmv) }\end{array}$ \\
\hline 1, 3 Butadiene & $106-99-0$ & 20,000 & 4,000 & 2,000 & 1000 \\
\hline Chloroform & $67-66-3$ & N/A & N/A & 500 & 250 \\
\hline 1, 4 Dioxane & $123-91-1$ & 20,000 & 4,000 & 500 & 250 \\
\hline Benzene & $71-43-2$ & 12,000 & 2,400 & 500 & 250 \\
\hline Carbon tetrachloride & $56-23-5$ & N/A & N/A & 200 & 100 \\
\hline Dichloromethane (Methylene chloride) & $75-09-2$ & 130,000 & 26,000 & 2,300 & 1,150 \\
\hline Acetaldehyde & $75-07-1$ & 40,000 & 8,000 & 2,000 & 1,000 \\
\hline Perchloroethylene & $127-18-4$ & N/A & N/A & 150 & 75 \\
\hline Ammonia & $7664-41-7$ & 150,000 & 30,000 & 300 & 150 \\
\hline Tributyl phosphate & $126-73-8$ & N/A & N/A & 30 & 15 \\
\hline Dibutyl phosphate & $107-66-4$ & N/A & N/A & 30 & 15 \\
\hline Hexane & $110-54-3$ & 12,000 & 2,400 & 1,100 & 550 \\
\hline Nitric oxide & $10102-43-9$ & N/A & N/A & 100 & 50 \\
\hline Acetone & $67-64-1$ & 25,000 & 5,000 & 2,500 & 1,250 \\
\hline n-Butanol & $71-36-3$ & 14,000 & 2,800 & 1,400 & 700 \\
\hline Nitrous oxide & $10024-97-2$ & N/A & N/A & N/A & N/A \\
\hline 3-Heptanone (Ethyl butyl ketone) & $106-35-4$ & N/A & N/A & 1,000 & 500 \\
\hline Nonane & $111-84-2$ & 8,500 & 1,700 & N/A & N/A \\
\hline 4-Heptanone (Dipropyl ketone) & $123-19-3$ & N/A & N/A & N/A & N/A \\
\hline n-Heptane & $142-82-5$ & 10,500 & 2,100 & 750 & 375 \\
\hline TNMHC & N/A & N/A & N/A & N/A & N/A \\
\hline
\end{tabular}

Note:

$\mathrm{N} / \mathrm{A}=$ not applicable or not available 
Table 3-3. Required Analytes: Program Requested Analytes/Selected Compounds Observed in Tank 241-C-106 Vapor or Waste Samples

\begin{tabular}{|c|c|c|c|c|c|}
\hline Analyte & $\begin{array}{c}\text { CAS } \\
\text { Number }\end{array}$ & $\begin{array}{c}\text { LFL } \\
\text { (ppmv) }\end{array}$ & $\begin{array}{c}20 \% \\
\text { LFL } \\
\text { (ppmv) }\end{array}$ & $\begin{array}{l}\text { DDLH } \\
\text { (ppmy) }\end{array}$ & $\begin{array}{l}50 \% \\
\text { IDLH } \\
\text { (ppmv) }\end{array}$ \\
\hline Methane & $74-82-8$ & 50,000 & 10,000 & N/A & N/A \\
\hline Ethane & $74-84-0$ & 30,000 & 6,000 & N/A & $\mathrm{N} / \mathrm{A}$ \\
\hline Ethylene & $74-85-1$ & 30,000 & 6,000 & N/A & $\mathrm{N} / \mathrm{A}$ \\
\hline 1-Hexene & $592-41-6$ & $\mathrm{~N} / \mathrm{A}$ & $\mathrm{N} / \mathrm{A}$ & $\mathrm{N} / \mathrm{A}$ & N/A \\
\hline Hexane & 110-54-3 & 12,000 & 2,400 & 1,100 & 550 \\
\hline 1-Heptene & $582-76-7$ & N/A & N/A & N/A & N/A \\
\hline 3-Heptene, (E)- & $14686-14-7$ & N/A & N/A & $\mathrm{N} / \mathrm{A}$ & N/A \\
\hline Heptane & $142-82-5$ & 10,500 & 2,100 & 750 & 375 \\
\hline 2-Heptene & $592-77-8$ & $\mathrm{~N} / \mathrm{A}$ & $\mathrm{N} / \mathrm{A}$ & N/A & N/A \\
\hline 3-Heptene & $292-78-9$ & $\mathrm{~N} / \mathrm{A}$ & N/A & N/A & N/A \\
\hline 2,4- Dimethyl-2,3-pentadiene & $1000-87-9$ & $\mathrm{~N} / \mathrm{A}$ & $\mathrm{N} / \mathrm{A}$ & N/A & N/A \\
\hline 2-Heptyne & $1119-65-9$ & $\mathrm{~N} / \mathrm{A}$ & N/A & N/A & $\mathrm{N} / \mathrm{A}$ \\
\hline 3-Methyl-heptane & $589-81-1$ & $\mathrm{~N} / \mathrm{A}$ & $\mathrm{N} / \mathrm{A}$ & N/A & N/A \\
\hline 3-Methylene-heptane & $1632-16-2$ & $\mathrm{~N} / \mathrm{A}$ & N/A & N/A & N/A \\
\hline 3-Methyl-3-heptene & $7300-03-0$ & N/A & $\mathrm{N} / \mathrm{A}$ & N/A & N/A \\
\hline 3-Ethyl-4-methyl-4-pentene & $61847-80-1$ & N/A & N/A & N/A & N/A \\
\hline 2-Octene & $111-67-1$ & N/A & N/A & N/A & N/A \\
\hline 4-Heptanone & $123-19-3$ & N/A & N/A & N/D & N/D \\
\hline 3-Heptanone & $106-35-4$ & N/A & N/A & 1,000 & 500 \\
\hline Nonane & $111-84-2$ & 8,500 & 1,700 & N/A & N/A \\
\hline Decane & 124-18-5 & 7,500 & 1,500 & $\mathrm{~N} / \mathrm{A}$ & $\mathrm{N} / \mathrm{A}$ \\
\hline Decahydro-naphthalene & 91-17-8 & 7,000 & 1,400 & N/A & N/A \\
\hline Undecane & $1120-21-4$ & N/A & N/A & N/A & $\mathrm{N} / \mathrm{A}$ \\
\hline Dodecane & $112-40-3$ & 6,000 & 1,200 & N/A & N/A \\
\hline Tridecane & $629-50-5$ & N/A & N/A & $\mathrm{N} / \mathrm{A}$ & N/A \\
\hline Tetradecane & $629-59-4$ & $\mathrm{~N} / \mathrm{A}$ & N/A & N/A & $\mathrm{N} / \mathrm{A}$ \\
\hline Pentadecane & $629-62-9$ & N/A & N/A & N/A & N/A \\
\hline
\end{tabular}

Note:

$\mathrm{N} / \mathrm{A}=$ not applicable or not available 
Table 3-4. Required Analytes: Target Analytes (2 sheets).

\begin{tabular}{|c|c|c|c|c|c|}
\hline Analyte & $\begin{array}{r}\text { CAS } \\
\text { Number } \\
\end{array}$ & $\begin{array}{l}\text { LFL } \\
\text { (ppmv) }\end{array}$ & $\begin{array}{l}20 \% \\
\text { LFL } \\
\text { (ppmv) }\end{array}$ & $\begin{array}{l}\text { IDLH } \\
\text { (ppmv) }\end{array}$ & $\begin{array}{l}50 \% \\
\text { DLH } \\
\text { (ppmv) }\end{array}$ \\
\hline Ammonia, $\mathrm{NH}_{3}$ & $7664-41-7$ & 150,000 & 30,000 & 300 & 150 \\
\hline Nitric oxide, NO & $10102-43-9$ & $\mathrm{~N} / \mathrm{A}$ & N/A & 100 & 50 \\
\hline Nitrogen dioxide, $\mathrm{NO}_{2}$ & 10102-44-0 & $\mathrm{N} / \mathrm{A}$ & $\mathrm{N} / \mathrm{A}$ & 20 & 10 \\
\hline Methane, $\mathrm{CH}_{4}$ & $74-82-8$ & 50,000 & 10,000 & N/A & N/A \\
\hline Carbon dioxide, $\mathrm{CO}_{2}$ & 124-38-9 & $\mathrm{N} / \mathrm{A}$ & N/A & 40,000 & 20,000 \\
\hline Carbon monoxide, $\mathrm{CO}$ & $630-08-0$ & 125,000 & 25,000 & 1,200 & 600 \\
\hline Nitrous oxide, $\mathrm{N}_{2} \mathrm{O}$ & $10024-97-2$ & $\mathrm{~N} / \mathrm{A}$ & N/A & N/A & N/A \\
\hline Hydrogen, $\mathrm{H}_{2}$ & $1333-74-0$ & 40,000 & 8,000 & N/A & N/A \\
\hline Acetaldehyde & $75-07-1$ & 40,000 & 8,000 & 2,000 & 1,000 \\
\hline Dichlorodifluoromethane (Freon 12) & 75-71-8 & $\mathrm{N} / \mathrm{A}$ & N/A & 15,000 & 7,500 \\
\hline Methyl chloride & $74-87-3$ & 81,000 & 16,200 & 2,000 & 1,000 \\
\hline n-Butane & $106-97-8$ & 18,000 & 3,600 & $\mathrm{~N} / \mathrm{A}$ & N/A \\
\hline Ethyl chloride & $75-00-3$ & 38,000 & 7,600 & 3,800 & 1,900 \\
\hline Ethanol & $64-17-5$ & 33,000 & 6,600 & 3,300 & 1,650 \\
\hline Trichlorofluoromethane (Freon 11) & $75-69-4$ & N/A & N/A & 2,000 & 1,000 \\
\hline Ethanenitrile (acetonitrile) & $75-05-8$ & 30,000 & 6,000 & 500 & 250 \\
\hline Propanone (acetone) & 67-64-1 & 25,000 & 5,000 & 2,500 & 1,250 \\
\hline Furan & $110-00-9$ & N/A & N/A & N/A & N/A \\
\hline n-Pentane & 109-66-0 & 14,000 & 2,800 & 1,500 & 750 \\
\hline 2-Propanol & $67-63-0$ & 20,000 & 4,000 & 2,000 & 1,000 \\
\hline Dichloromethane (me & $75-09-2$ & 130,000 & 26,000 & 2,300 & 1,150 \\
\hline 1-Propanol & $71-23-8$ & 22,000 & 4,400 & 800 & 400 \\
\hline 2-Methyl pentane & $107-83-5$ & $\mathrm{~N} / \mathrm{A}$ & $\mathrm{N} / \mathrm{A}$ & $\mathrm{N} / \mathrm{A}$ & N/A \\
\hline Propanenitrile & $107-12-0$ & 31,000 & 6,200 & $\mathrm{~N} / \mathrm{A}$ & N/A \\
\hline Butanal & $123-72-8$ & 14,000 & 2,800 & 1,400 & 700 \\
\hline 1-Hexene & $592-41-6$ & N/A & N/A & N/A & N/A \\
\hline 2-Butanone & 78-93-3 & 14,000 & 2,800 & 3,000 & 1,500 \\
\hline n-Hexane & $110-54-3$ & 12,000 & 2,400 & 1,100 & 550 \\
\hline Trichloromethane (chlo & $67-66-3$ & $\mathrm{~N} / \mathrm{A}$ & $\mathrm{N} / \mathrm{A}$ & 500 & 250 \\
\hline Tetrahydrofuran & 109-99-9 & 20,000 & 4,000 & 2,000 & 1,000 \\
\hline 1-Butanol & 71-36-3 & 14,000 & 2,800 & 1,400 & 700 \\
\hline Benzene & 71-43-2 & 12,000 & 2,400 & 500 & 250 \\
\hline $\begin{array}{l}\text { Tetrachloromethane (carbon } \\
\text { tetrachloride) }\end{array}$ & $56-23-5$ & $\mathrm{~N} / \mathrm{A}$ & $\mathrm{N} / \mathrm{A}$ & 200 & 100 \\
\hline 1-Butanenitrile & $109-74-0$ & 16,500 & 3,300 & $\mathrm{~N} / \mathrm{A}$ & N/A \\
\hline 3-Methyl hexane & $589-34-4$ & $\mathrm{~N} / \mathrm{A}$ & $\mathrm{N} / \mathrm{A}$ & $\mathrm{N} / \mathrm{A}$ & N/A \\
\hline
\end{tabular}


HNF-4030, Rev. 0

Table 3-4. Required Analytes: Target Analytes (2 sheets).

\begin{tabular}{|l|r|r|r|r|r|}
\hline \multicolumn{1}{|c|}{ Analyte } & $\begin{array}{r}\text { CAS } \\
\text { Number }\end{array}$ & $\begin{array}{r}\text { LFL } \\
\text { (ppmv) }\end{array}$ & $\begin{array}{r}\text { LFL } \\
\text { (ppmv) }\end{array}$ & $\begin{array}{r}\text { IDLH } \\
\text { (ppmv) }\end{array}$ & $\begin{array}{r}50 \% \\
\text { IDLH } \\
\text { (ppmv) }\end{array}$ \\
\hline 2-Pentanone & $107-87-9$ & 15,000 & 3,000 & 1,500 & 750 \\
\hline n-Heptane & $142-82-5$ & 10,500 & 2,100 & 750 & 375 \\
\hline 1,4-Dioxane & $123-91-1$ & 20,000 & 4,000 & 500 & 250 \\
\hline 4-Methyl-2-pentanone (hexone) & $108-10-1$ & 12,000 & 2,400 & 500 & 250 \\
\hline Toluene & $108-88-3$ & 11,000 & 2,200 & 500 & 250 \\
\hline 2-Hexanone & $591-78-6$ & N/A & N/A & 150 & 75 \\
\hline n-Octane & $111-65-9$ & 9,500 & 1,900 & 1,000 & 500 \\
\hline Tetrachloroethylene & $127-18-4$ & N/A & N/A & 150 & 75 \\
\hline Chlorobenzene & $108-90-7$ & 13,000 & 2,600 & 1,000 & 500 \\
\hline Ethylbenzene & $100-41-4$ & 8,000 & 1,600 & 800 & 400 \\
\hline m, p-Xylene & $106-42-3$ & 11,000 & 2,200 & 900 & 450 \\
\hline 3-Heptanone & $106-35-4$ & N/A & N/A & 1,000 & 500 \\
\hline 2-Heptanone & $110-43-0$ & 11,000 & 2,200 & 800 & 400 \\
\hline Cyclohexanone & $108-94-1$ & 11,000 & 2,200 & 700 & 350 \\
\hline Styrene & $100-42-5$ & 9,000 & 1,800 & 700 & 350 \\
\hline n-Nonane & $111-84-2$ & 8,500 & 1,700 & N/A & N/A \\
\hline o-Xylene & $95-47-6$ & 9,000 & 1,800 & 900 & 450 \\
\hline 1,1,2,2-Tetrachloroethane & $79-34-5$ & N/A & N/A & 100 & 50 \\
\hline 2-Octanone & $111-13-7$ & N/A & N/A & N/A & N/A \\
\hline n-Decane & $124-18-5$ & 7,500 & 1,500 & N/A & N/A \\
\hline 1,2,4-Trimethylbenzene & $95-63-6$ & 9,000 & 1,800 & N/A & N/A \\
\hline Not & & & &
\end{tabular}

Note:

$\mathrm{N} / \mathrm{A}=$ not applicable or not available 


\subsection{QUALITY ASSURANCE AND QUALITY CONTROL}

Vapor sampling and analysis shall be performed in accordance with approved quality assurance (QA) plans. These plans are required to meet the Hanford Analytical Services Quality Assurance Requirements Document (HASQARD) (DOE 1998) requirements. Validation of this compliance shall be verified either by a HASQARD assessment stating their quality program satisfactorily meets the appropriate requirements, or the quality program plan and applicable procedures will be submitted and approved prior to work performance on sampling or analytical work. Quality requirements for conducting Characterization Project sampling and analysis are described in Tank Waste Remediation System Characterization Project, Quality Policies (Board 1998) and this sampling and analysis plan. Characterization Project sampling and analysis shall be conducted in conformance with these QA requirements.

Processes, services, activities, and conditions adverse to quality which do not conform to requirements specified in this sampling and analysis plan or references herein shall be controlled to prevent inadvertent use. Nonconforming sampling and analysis processes shall be identified, controlled, reported, and dispositioned as required by PHMC (1998).

\subsection{LABORATORY OPERATIONS}

Analytical procedures that are to be used for this sample and analysis plan shall be written and approved prior to the performance of analytical work within the laboratory. Analytical QC requirements are identified in Table 3-1. The laboratory shall also use calibration and calibration check standards appropriate for the analytical instrumentation being used (see DOE [1998] for definitions of QC samples and standards). The criteria presented are goals for demonstrating reliable method performance. It is understood that the laboratory will follow its internal QC system for required actions whenever QC failures occur. If sample QC failures occur or if all analyses cannot be performed (e.g., insufficient sample), analysts shall consult with supervisors/customers to determine the proper action. The laboratory should provide a suggested course of action at that time. All sample QC failures and limitations on the associated data shall be discussed in the narrative of the data report. Proper notification of all data not meeting QC requirements shall be included with the data.

\subsection{SAMPLE COLLECTION}

Sampling shall be performed in accordance with approved procedures and work plans included within the job control system. All data sheets and log entries completed during the performance of sampling shall be copied and included within the job control system package.

Each sample identification number shall have the following format:

\section{VLXXX-YYY-ZZZZ}


where,

$\mathbf{V}$ indicates a vapor sample,

$\mathbf{L}=$ a letter code identifying the organization that prepared the sample container/sample media $\mathrm{C}=\mathrm{CPO}$ $\mathrm{S}=\mathrm{SAS}$,

$\mathbf{X X X}=$ a three-digit/letter code identifying the sample location $006=296-\mathrm{C}-006$ stack

$\mathrm{AMB}=$ ambient air samples at $\mathrm{C}$ tank farm $702=241-\mathrm{AZ}-702$ stack,

YYY = a three-digit sample code found in Tables 2-2, 2-3, and 2-4,

$\mathbf{Z Z Z Z}=$ a special lab-assigned or CPO-assigned code.

\subsection{SAMPLE CUSTODY}

Chain-of-custody will be carefully maintained to assure sample control at all times. 
HNF-4030, Rev. 0

\subsection{EXCEPTIONS, CLARIFICATIONS, AND ASSUMPTIONS}

\section{Trip Blanks and Field Blanks}

Trip Blanks are sampling devices prepared and handled in the same manner as samples, except that they are never opened in the field. Field Blanks are sampling devices prepared and handled in the same manner as the samples, but no tank gases are drawn through them. 
HNF-4030, Rev. 0

\subsection{ORGANIZATION}

The organization and responsibility of key personnel involved with these tank characterization projects are listed in Table 6-1.

Table 6-1. Project Key Personnel

\begin{tabular}{|c|c|c|}
\hline Responsibility & Organization & Individual \\
\hline $\begin{array}{l}\text { Data Assessment and } \\
\text { Interpretation manager }\end{array}$ & $\begin{array}{l}\text { TWRS Process Engineering } \\
\text { (LMHC) }\end{array}$ & K. M. Hall, 376-5029 \\
\hline $\begin{array}{l}\text { Process Engineering point of } \\
\text { contact for vapor sampling }\end{array}$ & $\begin{array}{l}\text { TWRS Process Engineering } \\
\text { (LMHC) }\end{array}$ & L. M. Sasaki, 373-1027 \\
\hline $\begin{array}{l}\text { Waste retrieval sluicing } \\
\text { system operations manager }\end{array}$ & $\begin{array}{l}\text { Retrieval Operations } \\
\text { Program (NHC) }\end{array}$ & J. W. Lentsch, 373-5252 \\
\hline $\begin{array}{l}\text { Waste retrieval sluicing } \\
\text { system technical contact }\end{array}$ & $\begin{array}{l}\text { TWRS Process Engineering, } \\
\text { (LMHC) }\end{array}$ & K. G. Carothers, 373-4556 \\
\hline $\begin{array}{l}\text { Vapor sampling cognizant } \\
\text { engineer }\end{array}$ & $\begin{array}{l}\text { Characterization Field } \\
\text { Engineering (LMHC) }\end{array}$ & D. D. Wanner, 373-3297 \\
\hline $\begin{array}{l}\text { SAS vapor sampling and } \\
\text { analysis project manager }\end{array}$ & $\begin{array}{l}\text { Special Analytical Support } \\
\text { (NHC) }\end{array}$ & L. L. Lockrem, 373-4771 \\
\hline $\begin{array}{l}\text { SAS vapor sampling and } \\
\text { analysis technical contact }\end{array}$ & $\begin{array}{l}\text { Special Analytical Support } \\
\text { (NHC) }\end{array}$ & R. S. Viswanath, 376-9223 \\
\hline $\begin{array}{l}\text { Industrial Hygiene and } \\
\text { Safety points of contact }\end{array}$ & $\begin{array}{l}\text { Tank Farm Facilities } \\
\text { Operations, Field Safety } \\
\text { Services (LMHC) }\end{array}$ & $\begin{array}{l}\text { K. M. Bowen, 372-3667 } \\
\text { N. K. Butler, 376-5795 }\end{array}$ \\
\hline $\begin{array}{l}\text { Double-Shell Tank Farm } \\
\text { point of contact }\end{array}$ & Tank Farm Operations & $\begin{array}{l}\text { Double-Shell Tank Farm } \\
\text { Operations shift manager, } \\
373-2689\end{array}$ \\
\hline $\begin{array}{l}\text { Senior Supervisory Watch, } \\
\text { MO-211 }\end{array}$ & $\begin{array}{l}\text { C-106 Sluicing Operations } \\
\text { (LMHC) }\end{array}$ & G. N. Hanson, 376-2182 \\
\hline $\begin{array}{l}\text { Test Director, Waste } \\
\text { Retrieval Sluicing System } \\
\text { Process Test }\end{array}$ & $\begin{array}{l}\text { C-106 Sluicing Operations } \\
\text { (LMHC) }\end{array}$ & K. J. Anderson, 373-6039 \\
\hline $\begin{array}{l}\text { Environmental point of } \\
\text { contact }\end{array}$ & $\begin{array}{l}\text { Environmental } \\
\text { Permits/Policy (LMHC) }\end{array}$ & G. M. Crummel, 373-5175 \\
\hline
\end{tabular}




\subsection{DELIVERABLES}

Sampling and analytical results shall be reported as Format VI reports. Any analyte exceeding the notification limit prescribed in Table 3-1 shall also be reported as a Format I report. In addition, Format II reports shall be provided by SAS, CPO, and WSCF as described in Section 7.2 .

\subsection{FORMAT I REPORTING}

Table 3-1 contains the notification limits for specific analytes. Analytes that exceed notification limits shall be reported by the Project Manager or delegate by calling the DoubleShell Tank Farms Operations shift manager as soon as the data are obtained and reviewed by the responsible scientist. This verbal notification must be followed within one hour by electronic notification to the Double-Shell Tank Farms Operations shift manager, the Industrial Hygiene and Safety point of contact, the TWRS Process Engineering Data Assessment and Interpretation manager, the waste retrieval sluicing system point of contact and the Process Engineering point of contact for vapor sampling. A further review of the data, including quality control results and additional analyses for verification purposes may be contracted with the performing laboratory by either a revision to this sampling and analysis plan or by a letter.

\subsection{FORMAT II REPORTING}

WSCF shall provide the results of radiological analyses using the standard WSCF analytical laboratory report format. The results shall be faxed to SAS and the Process Engineering vapor sampling point of contact within 48 hours of receipt of the samples and followed by transmittal of a copy of the results via plant mail.

CPO shall provide information on its sampling activities (copies of sampling data sheets, J-5 forms, chain of custody forms, and other pertinent documentation) to SAS and the Process Engineering vapor sampling point of contact. The information provided shall include: the sample collection sequence and volumes, start and stop times for the collection of each sample, the TOC reading at the start of the collection of each sample, and any anomalous sampling conditions. This information shall be provided within 48 hours of the collection of the last set of samples.

The SAS sampling team shall provide information on its sampling activities to the Process Engineering vapor sampling point of contact. The information provided shall include: the sample collection sequence and volumes, start and stop times for the collection of each sample, the TOC reading at the start of the collection of each sample, verification of trip and field blank use, and any anomalous sampling conditions. This information shall be provided within 48 hours of the collection of the last set of samples. 


\subsection{FORMAT VI REPORTING}

The Format VI report shall consist of two deliverables, preliminary analytical results and a final data package.

Preliminary sampling and analytical data shall be delivered within two weeks of the receipt of the samples at the laboratory. The preliminary data shall consist of, at a minimum, data tables reporting sample collection data, particulate filter analysis results, and the results of each analysis performed by the analytical laboratory. The following individuals shall be on distribution for the preliminary results: J. W. Bailey, N. K. Butler, K. G. Carothers, G. M. Crummel, J. W. Lentsch, R. L. Powers, and L. M. Sasaki.

A data package shall be issued as a supporting document within seven weeks of the receipt of the samples at the laboratory. The data package shall contain the elements listed in Table 7-1. The following individuals shall be on distribution for the entire data package: R. A. Bechtold, G. M. Crummel, and L. M. Sasaki. The following individuals require only the engineering data transmittal: J. W. Bailey, K. G. Carothers, J. W. Lentsch, N. K. Butler, R. L. Powers, and C. A. Simonen.

In addition to the data package, an electronic version of the analytical results shall be provided to the Tank Vapor Database representative within 4 calendar days from the day that the final data package is issued. The data must be available to the Washington State Department of Ecology within 7 calendar days of release of the data package. The electronic version shall be in the standard electronic format specified in Bobrowski and Simonen (1999).

Table 7-1. Data Package Required Elements ( 2 sheets). ${ }^{1}$

\begin{tabular}{|l|}
\hline \multicolumn{1}{|c|}{ Prefatory Elements } \\
\hline Executive summary \\
\hline Table of contents \\
\hline List of abbreviations and acronyms \\
\hline Quality assurance data package review results \\
\hline Non-conformance reports \\
\hline
\end{tabular}


HNF-4030, Rev. 0

Table 7-1. Data Package Required Elements (2 sheets). ${ }^{1}$

Sampling Elements

Sampling case narrative

Sample summary and event chronology

Sampling procedures table

Sampling logbook table

Field data

Radiation screening results

Chain of custody forms

Analysis Elements

Analytical case narrative

Analytical procedures table

Data qualifier flag translation table

Target analytes concentration table

Tentatively identified compound concentration table

Laboratory blank summary

Field blank summary

Trip blank summary

Mass spectrometer instrument tune report

Target analyte initial calibration table

Internal standards area counts table

Laboratory control sample results table

Surrogate compounds results table

Quantitation reports

Chromatograms

Mass spectra of reported tentatively identified compounds

Note:

${ }^{1}$ Include all elements as applicable. 
HNF-4030, Rev. 0

\subsection{CHANGE CONTROL}

Under certain circumstances, it may become necessary for the performing laboratory to make decisions concerning a sample without review of the data by the customer or the Characterization Project. All significant changes shall be documented by TWRS Process Engineering via an engineering change notice to this sampling and analysis plan or by a letter. All changes shall also be clearly documented in the final data report. Insignificant changes may be made by placing a notation in the permanent record (i.e., note change in log book or memo to file). Significance is determined by the Process Engineering point of contact for vapor sampling.

At the request of the Retrieval Operations Project, additional analysis of sample material from this characterization project shall be performed following a revision of this sampling and analysis plan or issuance of a letter. 
HNF-4030, Rev. 0

\subsection{REFERENCES}

Board, D. C., 1998, Tank Waste Remediation System, Characterization Project, Quality Policies, HNF-SD-WM-QAPP-025, Rev. 4, Lockheed Martin Hanford Corp. for Fluor Daniel Hanford, Inc., Richland, Washington.

Bobrowski, S. F., and C. A. Simonen, 1999, Standard Electronic Format Specification for Tank Vapor Data MSEXCEL Spreadsheets: Version 1.0, HNF-3815, Rev. 0, prepared by Pacific Northwest National Laboratory for Lockheed Martin Hanford Corp, Richland, Washington.

DOE, 1998, Hanford Analytical Services Quality Assurance Requirements Document, DOE/RL-96-68, Rev. 2, U.S. Department of Energy, Richland Field Office, Richland, Washington.

Jones, J. M., 1999, Waste Retrieval Sluicing System Emissions Collection Phase II, PTP-320001, Rev. A-1, Lockheed Martin Hanford Corp. for Fluor Daniel Hanford, Inc., Richland, Washington.

Mulkey, C. H., and K. D. Markillie, 1995, Data Quality Objectives for Regulatory Requirements for Hazardous and Radioactive Air Emissions Sampling and Analysis, WHC-SDWM-DQO-021, Rev. 0, Westinghouse Hanford Company, Richland, Washington.

Peterson, K. A., 1998, Request for Ecology Concurrence on Draft Strategy/Path Forward to Address Concerns Regarding Organic Emissions from C-106 Sluicing Activities, (Meeting Minutes, December 3), Fluor Daniel Hanford, Inc., Richland, Washington.

PHMC, 1998, Nonconforming Item Reporting and Control, HNF-PRO-298, Rev. 1, Fluor Daniel Hanford, Inc. Richland, Washington.

Powers, R. L., 1999, Process Test Plan, Phase II: Waste Retrieval Sluicing System Emissions Collection, HNF-4034, Rev. 0, Lockheed Martin Hanford Corp. for Fluor Daniel Hanford, Inc., Richland, Washington. 


\section{DISTRIBUTION SHEET}

\begin{tabular}{|c|c|c|c|c|c|}
\hline \multirow{2}{*}{$\begin{array}{l}\text { To } \\
\quad \text { Distribution }\end{array}$} & \multirow{2}{*}{\multicolumn{3}{|c|}{ From }} & \multicolumn{2}{|l|}{ Page 1 of 2} \\
\hline & & & & \multicolumn{2}{|c|}{ Date $\quad 02 / 12 / 99$} \\
\hline \multicolumn{4}{|l|}{ Project Title/Work Order } & \multicolumn{2}{|c|}{ EDT No. EDT-611450 } \\
\hline \multicolumn{4}{|c|}{$\begin{array}{l}\text { HNF-4030. Rev. 0. "Waste Retrieval Sluicing System Vapor } \\
\text { Sampling and Analysis Plan for Evaluation of Organic Emissions, } \\
\text { Process Test Phase II" }\end{array}$} & \multicolumn{2}{|l|}{ ECN No. $\quad N / A$} \\
\hline Name & MSIN & $\begin{array}{l}\text { Text } \\
\text { With } \\
\text { Al } 1 \\
\text { Attach. }\end{array}$ & Text only & $\begin{array}{l}\text { Attach./ } \\
\text { Appendix } \\
\text { Only }\end{array}$ & $\begin{array}{l}\text { EDT/ECN } \\
\text { Only }\end{array}$ \\
\hline
\end{tabular}

U. S. Department of Energy -

Richland Field Office

W Abdul

DOE/RL Reading Room

S7-54 $\quad X$

H2-53 X

COGEMA

J. R. Bellomy

L. A. Pingel

S5-05 $\quad x$

S3-90 $\quad x$

R. S. Viswanath

S3-90 $\quad x$

Fluor Daniel Northwest

D. L. Evans

S2-47 $\quad x$

Lockheed Martin Hanford Corp.

D. C. Board

K. M. Bowen

$57-07$

$55-12$

S7 -12

R. G. Brown

R. L. Brown

S6-14

W. E. Bryan

S5- 05

N. K. Butler

K. G. Carothers

S5- 12

R2-11

G. M. Crummel

K. M. Hall

G. N. Hanson

R1-51

R2 -12

S5-07

R? - 11

R1-51

C. H. Mulkey

R. L. Powers

S5-13

R2-12

L. M. Sasaki

L. A. Stauffer

R2 - 11

D. D. Wanner

T.C.S.R.C.

S7 -12

R1-10

$x$
$x$

Lockheed Martin Services, Inc.

Central Files

B1-07

$$
\begin{aligned}
& x \\
& x \\
& x \\
& x \\
& x \\
& x \\
& x \\
& x \\
& x \\
& x \\
& x \\
& x \\
& x \\
& x \\
& x \\
& x \\
& x
\end{aligned}
$$$$
X
$$

$\hat{x}$

$x$
$x$

$x$

Los Alamos Technical Associates

J. M. Jones

$55-13 \quad x$

MacTec

J. C. Guyette

S7 $-40 \quad x$




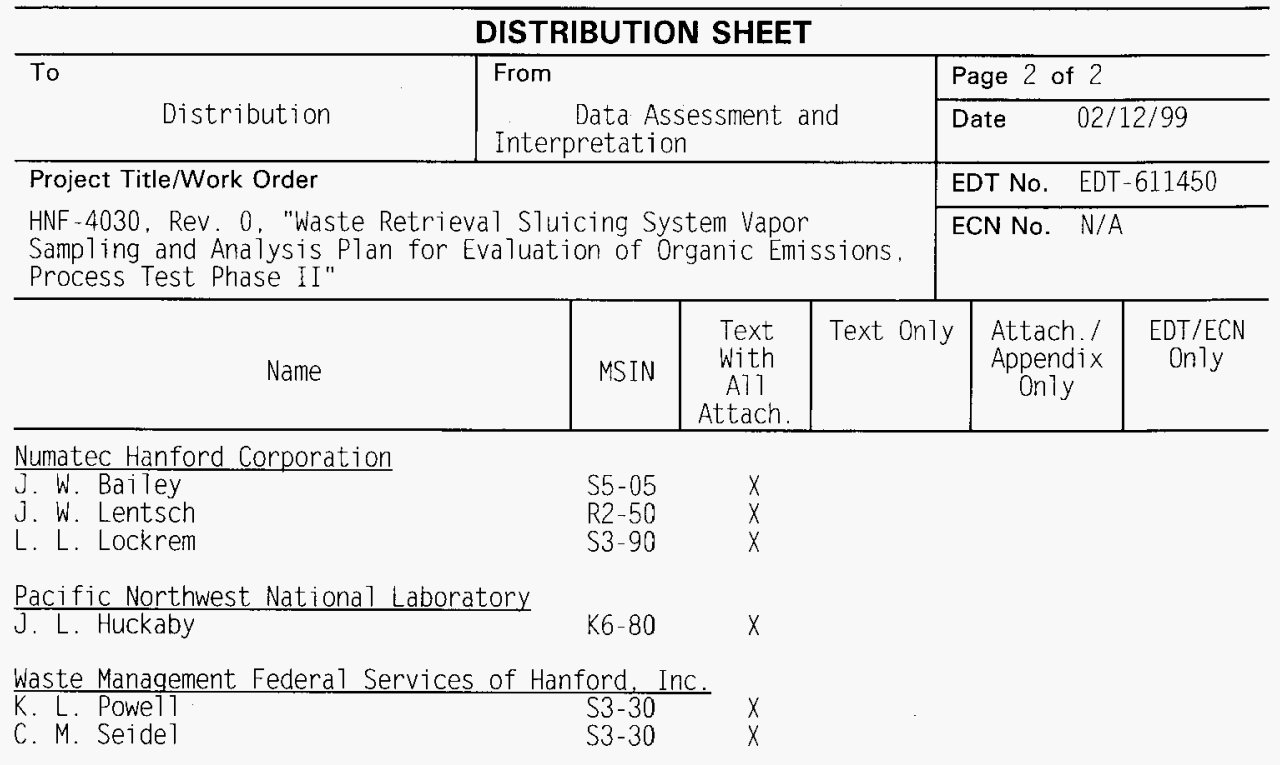

